\title{
(Sub) Optimality and (Non) Optimal Satisficing in Risky Decision Experiments
}

\author{
Daniela Di Cagno ${ }^{* 1}$, Arianna Galliera ${ }^{\dagger 1}$, Werner Güth ${ }^{\ddagger 1,2,3}$, Francesca Marzo ${ }^{\S 1}$ and \\ Noemi Pace 4 \\ ${ }^{1}$ Luiss, Rome \\ ${ }^{2}$ Max Planck, Bonn \\ ${ }^{3}$ Frankfurt Business School \\ ${ }^{4}$ University of Venice, Ca' Foscari
}

November 24, 2016

\begin{abstract}
We implement a risky choice experiment based on one-dimensional choice variables and risk neutrality induced via binary lottery incentives. Each participant confronts many parameter constellations with varying optimal payoffs. We assess (sub)optimality, as well as (non)optimal satisficing by eliciting aspirations in addition to choices. Treatments differ in the probability that a binary random event, which are payoff- but not optimal choice-relevant, is experimentally induced and whether participants choose portfolios directly or via satisficing, i.e., by forming aspirations and checking for satisficing before making their choice. By incentivizing aspiration formation, we can test satisficing, and in cases of satisficing, determine whether it is optimal.
\end{abstract}

Keywords: (un)Bounded Rationality, Satisficing, Risk, Uncertainty, Experiments

JEL: D03; D81; C91

\section{Introduction}

The rational choice approach, albeit dominating (micro)economics, should be considered with caution in the real world because optimizing is often difficult: limited cognitive abilities, information

\footnotetext{
*ddicagno@luiss.it

†agalliera@luiss.it

${ }^{\ddagger}$ gueth@coll.mpg.de

§marzo@luiss.it

『n.pace@unive.it

The research presented in this paper was financed by the Max Planck Institute of Bonn.
} 
overload and complexity will regularly lead to suboptimal decision making. ${ }^{1}$ Such regular suboptimality renders also "rationality with errors" questionable. ${ }^{2}$ Moreover most choice situations involve multiple incompatible goals that must somehow be combined to reach a decision (as in multiobjective optimization). ${ }^{3}$

Many scholars now focus on alternative models ${ }^{4}$ and, more generally, on bounded rationality ${ }^{5}$, such as satisficing behavior. To compare these two different strands of the literature (i.e., optimizing versus satisficing), we implement a choice class allowing not only for unique and set-valued optimality in the sense of expected utility maximization but also experimentally elicit and incentivize aspiration formation. Although the experimental setup is framed as a financial investment task this is only done to discourage other regarding concerns.

Our main aim in designing it has been to control the usual tool used to align rational choice predictions with choice behavior. How this can be achieved will be described when presenting the class of experimental choice task. What can be said already now is that we do not claim external validity for the choice tasks. Rather they were signed to allow clearcut tests of optimality as well as optimal satisficing in consequentialistic decision making. ${ }^{6}$ Our main message is to illustrate that, even when rationality relies on rather mild assumptions both optimality and satisficing might require experience. ${ }^{7}$ In particular, in our setting each participant confronts two random sequences of 18 different (continuous) choice tasks whose expected success depends on a binary random event. Optimality requires only to prefer more money to less and to find the focal (corner) maximum of the expected success with several trials. Thus optimality can be assessed based on how choices deviate from the (corner) optimum and how costly this is.

To avoid criticizing without providing alternatives, we also consider bounded rationality based

\footnotetext{
${ }^{1}$ See Buchanan and Kock (2001) on information overload issues.

${ }^{2}$ For an example of this literature, see Hey and Orme (1994), Hey (1995), Loomes and Sugden (1995), and Harless and Camerer (1994), among others.

${ }^{3}$ Marley (1997).

${ }^{4}$ See, among others, Savikhin (2013) on financial analysis and risk management.

${ }^{5}$ See, for instance, Selten et al. (2012) and Güth and Ploner (2016)

${ }^{6}$ Consequentialist bounded rationality assumes that choosing among alternatives by anticipating their likely implications requires causal relationships linking the choice (means) and determinants beyond one's control, such as chance events, to the relevant outcome variables (ends).

${ }^{7}$ Note that this kind of experimental analysis can shed light on mental modeling and - more generally - on cognitive processes, in addition to eliciting the usual choice data.
} 
on consequentialist choice deliberations and satisficing rather than optimizing. Instead of reacting to given and well-behaved preferences and beliefs about circumstances beyond their control, participants are asked to form goal aspirations and then successively test behavioral options to determine whether they are satisficing these aspirations before making a choice. From a methodological perspective this means that we do not rely on the revealed aspiration approach for empirical economics. Whereas "revealed motives" presuppose satisficing (respectively optimizing) eliciting aspirations in addition to choice data allows to test satisficing. ${ }^{8}$

In our setup, the realization of a binary chance event is beyond our control either "boom" (good outcome) or "doom" (bad outcome) circumstances result. Abstaining from imposing intrapersonal payoff aggregation as in expected utility and prospect theory (by aggregating the probability-weighted choice implications in boom and doom), means that participants form goal aspirations for boom and doom scenarios. When satisficing, one chooses a portfolio whose returns in boom, respectively doom, satisfy both aspirations. This does not rule out optimality as a border case: set-valued optimal satisficing requires that it is impossible to increase the aspiration for boom or doom without reducing the other. Such set optimality does not require probabilities of boom and doom, which are partly not experimentally induced. ${ }^{9}$ Even when probabilities are experimentally induced, they may not be used for intrapersonal payoff aggregation but in forming and adapting aspiration levels, for instance, by forming more ambitious (moderate) aspirations for the more (less) likely event.

A portfolio choice may be either satisficing (but not set optimal) or non-satisficing. In an experiment, one can confirm a portfolio choice even when it is not satisficing. According to our interpretation, satisficing is based on a forward-looking decision-making process involving several successive steps for the task at hand, aspiration formation and searching for satisficing options in the action space, with possible feedback loops in light of new information. We experimentally compare participants who are forced to reason according to this structure before deciding to participants who are allowed to decide freely, that is, without having to form aspirations. We neither predicted optimality

\footnotetext{
${ }^{8}$ From the seminal contribution of Simon (1955) to contributions in mathematics (see Kunreuther and Krantz, 2007) and psychology (Kruglanski 1996 and Kruglanski et al. 2002), as well as to the literature on the role of mental models in decision making (Gary and Wood, 2010), this approach has increasingly contaminated economics (Camerer 1991, Pearl 2003 and Gilboa and Schmeidler 2001), although not always beyond lip service.

${ }^{9}$ One essentially employs a multiple selves approach that does not require intrapersonal payoff aggregation.
} 
nor optimal satisficing and we expected even considerable non-satisficing choices that will become less frequent when participants become more experienced that would illustrate how experience can improve behavior.

Section 2 introduces the 18 choice tasks, or cases, and derives their optimal choices or choice sets. We then discuss the hypotheses in Section 3. The treatments and other details of the experimental protocol are described in Section 4. Sections 5 presents the results on (sub)optimality as pairwise comparisons of treatments; Section 6 focuses on satisficing and its statistical analysis, while Section 7 refers to special cases in the data. Section 8 concludes. The appendices include additional data analysis and the translated instructions of the experiment.

\section{The choice class}

To induce risk neutrality, participants earn, in addition to their show-up fee, either $€ 4$ or $€ 14$, i.e., we implement binary lottery incentives. In doing so, we are not troubled by doubtful evidence ${ }^{10}$ (see Selten et al., 1999) that such incentives imply risk neutrality. When testing expected utility theory, one presupposes that "binary lottery incentives work". Specifically, participants should maximize via their portfolio choice the probability of receiving $€ 14$ rather than $€ 4$, what assumes:

Assumption 1 Participants prefer more money, €14, to less, €4.

When describing the choice class, the financial portfolio selection frame, used in the instructions, does not claim external validity, i.e. to capture portfolio choice problems in the field. The choice class is designed to induce, for example, risk neutrality and to render the probabilities for boom and doom irrelevant for optimal choice. Furthermore, by framing the decision tasks as portfolio choices we try to strengthen the purely individual choice consequences, i.e., to discourage other-regarding concerns.

In each choice task an endowment (of a positive amount) $e$ can be invested in a risk-free bond with a constant repayment rate $c(\geq 0)$ or in a risky asset. The repayment rate of the risky asset is

\footnotetext{
${ }^{10}$ Of course, one may object that risk attitude can be interpreted much more broadly than captured by curvature of utility-of-money curves, e.g. via probability transformation. Again this we can say that most of other risk aspects seem to also play no or at best a minor role in our setup.
} 
$r(i)$ with probability $p$, where $r(i)$ depends on the amount $i$ invested in the risky asset, or it is 0 with probability $(1-p)$, where $0<p<1$. We refer to the $(1-p)$-probability event when $i$ is lost as doom and to the $p$-probability event as boom, where the return from $i$ is $r(i) \cdot i$. We let $r(i)$ depend linearly on $i$ via $r(i)=e-i$ for all $0 \leq i \leq e$.

$P(i)$ denotes the expected probability of earning $€ 14$ rather than $€ 4$. For the expected utility of choice $i$, namely, $E(i)=[1-P(i)] u(€ 4)+P(i) u(€ 14))$, setting $u(€ 4)=0$ and $u(€ 14)=$ 1 based on Assumption 1 implies that $E(i)=P(i)$, i.e., the expected utility of choice $i$ is the probability that choice $i$ implies for earning $€ 14$.

Now the return from investing $i$ in the risky asset and $e-i$ in the safe bond is $(e-i) c$ in case of doom and $(e-i)(c+i)$ in case of boom. Since $P(i)$ is restricted to $0 \leq P(i) \leq 1$, expected utility is given by:

$$
P(i)=(1-p) \cdot \min \{1,(e-i) c\}+p \cdot \min \{1,(e-i)(c+i)\}
$$

Across all cases, one has $(e-i) c<1$ via $e c<1$ due to $(e-i)(c+i) \leq 1$. Thus, the constrained optimization tasks require us to determine the $i$ level(s) for which

$$
P(i)=(1-p)(e-i) c+p \cdot \min \{1,(e-i)(c+i)\}
$$

is maximal. Solving the unconstrained maximization of

$$
P^{\circ}(i)=(1-p)(e-i) c+p(e-i)(c+i)
$$

yields $i^{\circ}=\frac{p e-c}{2 p}$, exceeding, for all cases with $c>0$, the smallest $i$ level for which $(e-i)(c+i)$ equals $1^{11}$, namely,

$$
i^{*}=\frac{e-c}{2}-\frac{\sqrt{(e+c)^{2}-4}}{2}
$$

Assumption $2{ }^{12}$ One can determine for $c>0$ the lowest $i$ level $i^{*}$ with $\min \{1,(e-i)(c+i)\}=1$.

\footnotetext{
${ }^{11}$ see Table 1 presenting the optimal predictions

${ }^{12}$ To justify Assumption 2 participants can consider 6 options $i$ by moving a slider displaying $\bar{P}(i)=$ $\min \{1,(e-i)(c+i)\}$ and $\underline{P}(i)=(e-i) c$ before making their choice, as it will be explained in the Experimental Protocol section
} 
We define $\bar{P}(i)=\min \{1,(e-i)(c+i)\}$ and $\underline{P}(i)=(e-i) c . \bar{P}(i)$ is the column height for boom and $\underline{P}(i)$ for doom that participants can see as resulting from a chosen slider position.

For all $i$ levels above $i^{*}$, especially for $i^{\circ}$, one can reduce $i$ and, as $c>0$, improve the chances of earning $€ 14$ in the doom case without reducing the chances of earning $€ 14$ with probability 1 in the boom case, as illustrated in the left diagram of Figure 1 with two curves representing $\bar{P}(i)$ and $\underline{P}(i)$. The left diagram in Figure 1 highlights that when lowering $i$ from $i=e$ one first improves both success probabilities, $\bar{P}(i)$ and $\underline{P}(i)$. Then when $\bar{P}(i)$ is constrained to 1 , respectively $100 \%$, only $\underline{P}(i)$ is still increasing whereas $\bar{P}(i)=1$ when $i$ is further decreased. Only when $i<i^{*}$ one faces conflicting concerns $\bar{P}(i)$ and $\underline{P}(i)$ : reducing $i$ in this range one favors $\underline{P}(i)$ at the cost of $\bar{P}(i)$. The right diagram of Figure 1 tries to visualize the geometry of optimality and optimal satisficing. It presents five different curves: the two strictly concave curves both neglecting the constraint $\bar{P}(i)=\min \{1,(e-i)(c+i)\}$ of which the upper is the unconstrained boom payoff $(e-i)(c+i)$ and the lower is the unconstrained payoff $P^{\circ}(i)$ in equation (3). The other curves display the success probability $\bar{P}(i)$ for the boom (flat top), $\underline{P}(i)$ (linear) for the doom, already contained in the left diagram. Finally the probability-weighted sum $P(i), \bar{P}(i)$ and $\underline{P}(i)$ is piecewise linear and concave, and shows the expected success $P(i)$ of choice $i$ in equation (2) whose corner maximum is $i^{*}$, and the lowest $i$ level with $\bar{P}(i)=1$. Whereas optimality requires unambiguously the choice of $i^{*}$, optimal satisficing only excludes $i$-choices with $i>i^{*}$ : all $i$-choices in the range $0 \leq i \leq i^{*}$ do not allow to increase either $\bar{P}(i)$ or $\underline{P}(i)$ without reducing the other.

Proposition 1 For $c>0$, the optimal unique investment is given by $i^{*}$ in equation (4), which does not depend on $p$.

For $c=0$, there exist no chances in doom to win $€ 14$ rather than $€ 4$, as $\underline{P}(i)=(e-i) c=0$. Thus, also for $c=0$ probability $p$ is not optimal-choice relevant and the problem of intra-personal payoff aggregation does not arise. If $c=0$ and $e=2$, the unique optimal choice is $i^{*}=1$ so that $\frac{i^{*}}{e}=1 / 2$, often referred to as "Golden Mean". When $c=0$ and $e>2$, all investment choices $i^{*}$ in the range

$$
\frac{e-c}{2}-\frac{\sqrt{e^{2}-4}}{2} \leq i \leq \frac{e-c}{2}+\frac{\sqrt{e^{2}-4}}{2}
$$


are optimal.

Figure 2 graphically illustrates that $\bar{P}(i)=1$ and $P(i)=p \cdot \max \{1,(e-i) i\}=p$ are flat in that range. For $e=2$, the flat interval degenerates to a single tangential point with $\left(e-i^{*}\right) i^{*}=1$. Figure 2, based on two (of the three) $c=0$ cases with $e=2$ and $e=4$, displays the curves $\bar{P}(i)$ for $e=2$ with $\bar{P}(i)=1$ just for $i^{*}=1$ as well as for $e=4$ with a wide range of optimal investments $i^{*}$ in the generic interval (see equation 5). Of course, the $P(i)$ - curves for both cases would represent $P(i)=p \bar{P}(i)$ would be linear but display the same curvature.

Proposition 2 For $c=0$, the point and set values optimality coincide and predict a unique choice for $e=2$ but a generic interval prediction (equation 5) for $e>2$.

Figure 1: Illustration of payoff incentives $P(i), \bar{P}(i), \underline{P}(i)$
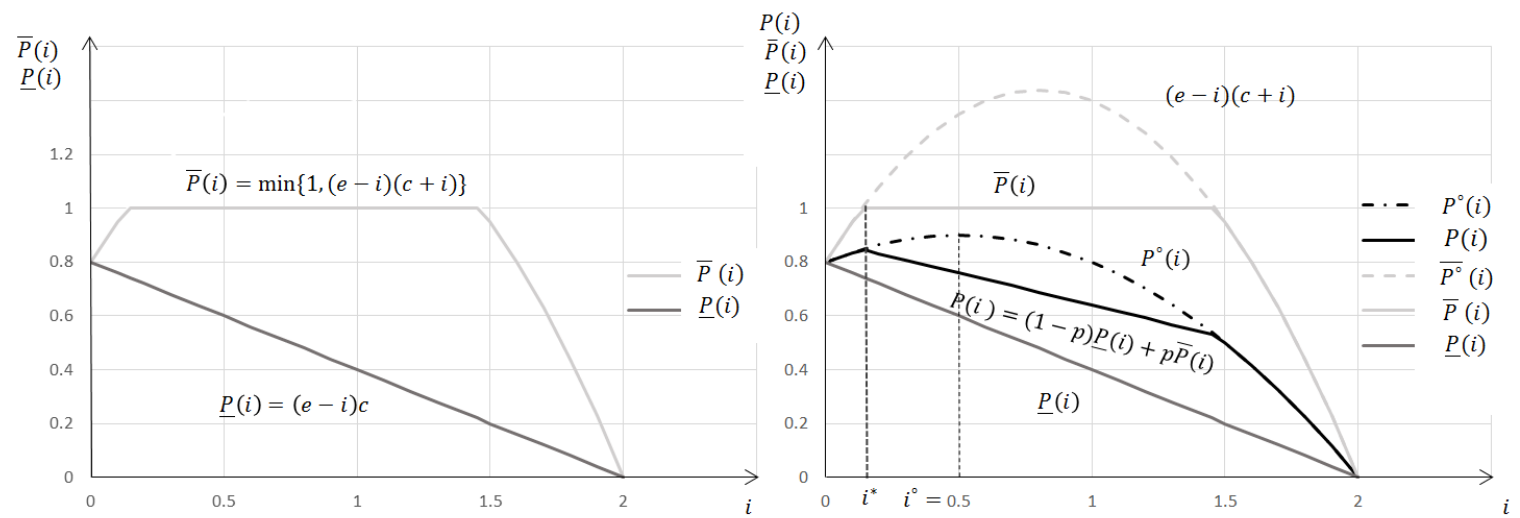

Notes: $p=c=0.4$ and $e=2$ yield a similar graph for all $p, c>0$ and $e \geq 2$ such that $0<i^{*}<i^{\circ}<\frac{e-c}{2}<\frac{e}{2}$.

We extend Assumption 2 to $c=0$ as follows:

Assumption 3 Participants can determine some $i$-level with $\bar{P}(i)=1$ when $c=0$.

Given that participants can use the slider repeatedly, this does not seem unrealistic for $e>2$ when the interval (equation 5) is generic. However, even for $e=2$, the unique optimal choice $i^{*}=1$ may be found after some experience. 
Figure 2: Illustration of payoff incentives $\bar{P}(i)$ when $c=0$ and $e=2$ or 4

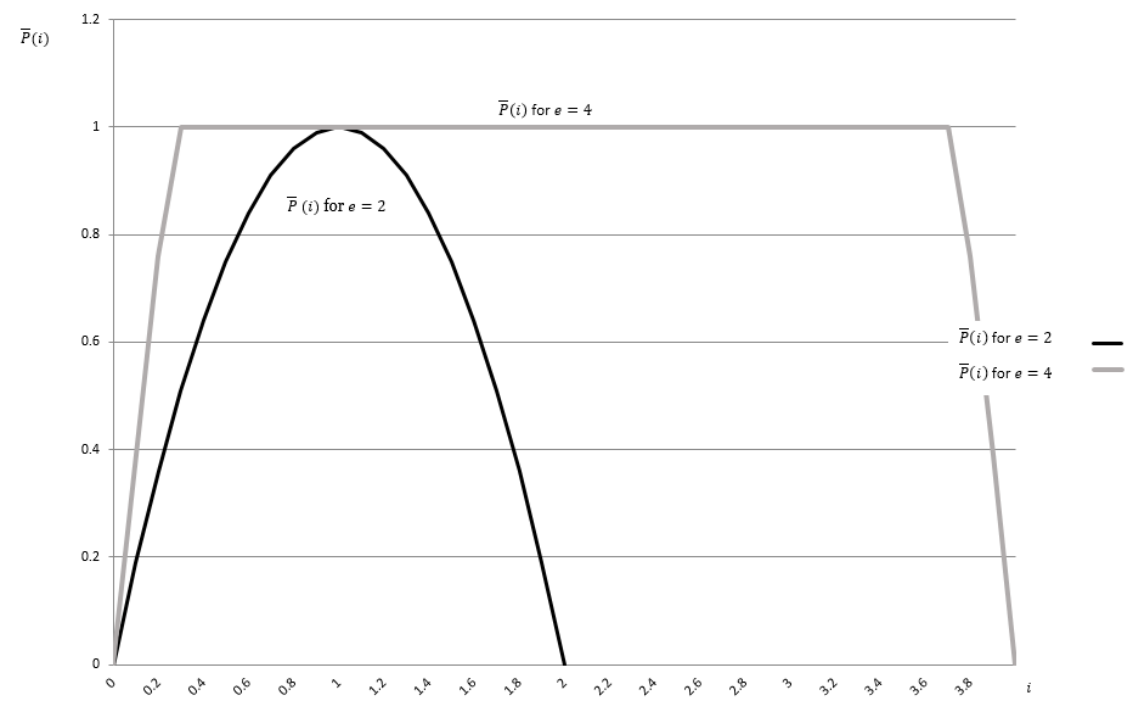

Notes: For $e=2$ and $e=4$.

The (sets of) optimal $i^{*}$ choices for all parameter constellations, referred to as cases, are listed in Table 3. When generating the parameter constellations confronted by participants, we wanted to include rather large and small probabilities $p$, although the numerical probability $p$ does not affect the optimal choice $i^{*}$. The advantage of our setting is that optimality can be achieved via the heuristic of determining the smallest $i$ level guaranteeing $\bar{P}(i)=1$ by repeatedly using the slider provided by the software (see the decision screens in Figures 9 and 10 in Appendix B and their explanation in the instructions in Appendix A).

\section{Hypotheses}

Each participant confronts all 18 different parameter constellations (twice) in two successive random orders. We refer to the first random sequence of 18 cases as phase 1 and to the second as phase 2 .

For (sub) optimality, one could predict symmetry ${ }^{13}$, as well as a decline with experience.

\footnotetext{
${ }^{13}$ In the spirit of Fechner's (1876) law for visual distance perception, symmetry could be questioned by concavity in perceiving numerical success for all numerical goals, irrespective whether the goals are monetary or probabilities of earning $€ 14$ rather than $€ 4$. Rather than risk aversion postulating a concave utility of money, in our context, the concavely perceived numerical goal would be the probability of earning $€ 14$, which suggests more $i$ choices below rather than above
} 
Hypothesis 1 (Symmetry and Learning Hypothesis): The observed $i$ choices are more symmetrically distributed below and above $i^{*}$ the closer $q^{*}=\frac{i^{*}}{e}$ is to $1 / 2$ with a variance of $i-i^{*}$ or $q-q^{*}=\frac{i}{q}-\frac{i^{*}}{q}$ being smaller in phase 2.

We do not analyze sub-optimality via "rationality in making mistakes" meaning that mistakes (here, deviations of $i$ from $i^{*}$ ) with higher losses - compared to optimality - are less likely. In our view, rationality in committing errors is questionable as it presupposes an awareness of what one loses. ${ }^{14}$

For $c>0$ for the concept of satisficing, aspiration formation means specifying an aspired probability

(1) $\bar{A}$ for $\bar{P}(i)=\min \{1,(e-i)(c+i)\}$

(2) $\underline{A}(\leq \bar{A})$ for $\underline{P}(i)=(e-i) c$ when the risky investment is lost.

Since $(e-i)(c+i)$ can partly exceed 1 for generic $i$ intervals, in these intervals, one can decrease the $i$ level and increase $\underline{P}(i)$ for $c>0$ without questioning $\bar{P}(i)=\min \{1,(e-i)(c+i)\}=1$. In the terminology of satisficing, one can form a higher doom aspiration, $\underline{A}$, without having to reduce the boom aspiration, $\bar{A}$.

Across all 15 cases with $c>0$, the optimal choice $i^{*}$ is the right corner of the interval from 0 to $i^{*}$ of all $i$ choices that are set optimal in the sense that it is impossible to increase $\bar{P}(i)$ without reducing $\underline{P}(i)$, and vice versa. Using the slider generates column heights indicating the probabilities of earning $€ 14$ in the boom and doom cases, which a participant has to translate into numerical success probabilities. This might cause problems when considering $i$ choices suggesting probability aspirations for $€ 14$ below 1 that, however, only apply to set-optimal choices $i$, with $i<i^{*}$ for $c>0$. For $i$ choices that guarantee earning $€ 14$ with probability 1 , the translation of column heights into numerical aspirations $\bar{A}=1$ should matter less.

If a choice $i$ with $0 \leq i \leq e$ is guaranteeing $\bar{P}(i) \geq \bar{A}$, as well as $\underline{P}(i) \geq \underline{A}$, we say that $i$ is satisficing $(\bar{A}, \underline{A})$. Furthermore, we speak of optimal satisficing if neither $\bar{A}$ nor $\underline{A}$ can be increased $i^{*}$.

\footnotetext{
${ }^{14}$ Nevertheless, concepts relying on "rational mistakes" are often used to account for empirical, mostly experimentally observed, behavior, e.g., Quantal Response (Equilibrium) estimates (McKelvey and Palfrey, 1995), but also in game theory, e.g., in case of the "intuitive criterion" (Cho and Kreps, 1987) and "properness" (Myerson, 1978).
} 
without questioning such satisficing. For $c>0$, optimal satisficing excludes any choice $i$ yielding $(e-i)(c+i)>1$ : if $i>i^{*}:=\frac{e-c}{2}-\frac{\sqrt{(e+c)^{2}-4}}{2}$, one can increase $\underline{P}(i)$ by a lower $i$ in the range $i^{*} \leq i$ without questioning that $\bar{P}(i)=1$. Since for the 15 cases with $c>0$ one has $e \geq 2$ and, thus, $(e+c)^{2}>4$, the term $\sqrt{(e+c)^{2}-4}$ is positive across all $c>0$ cases: set optimality in the sense of optimal satisficing requires $0 \leq i \leq i^{*}$ for $c>0$ (see Figure 1 for an illustration).

The set-optimal choices $i$ and aspiration profiles $(\bar{A}, \underline{A})$ with $\bar{A} \geq \underline{A}$ are thus given by $0 \leq i \leq i^{*}$, $\bar{A}=\bar{P}(i)=1$ and $\underline{A}=\underline{P}(i)=c(e-i)$. This set definition of optimally satisficing choices $i$ and of optimal aspiration profiles $(\bar{A}, \underline{A})$ does not pay any attention to probability $p$. Rather than weighting cases (1) and (2) by probabilities and aggregating their probability-weighted success, the decision maker is concerned with two alter egos, only one of which would be rewarded for risky investment.

For the three $c=0$ cases, the doom scenarios has $\underline{P}(i)=0$ for all $i$ with $0 \leq i \leq e$. Thus, one should only aspire $\underline{A}=0$, which avoids intra-personal payoff aggregation. As a consequence, the sets of utility-maximizing and optimally satisficing choices coincide with the point prediction $i^{*}=\frac{(e-c)}{2}$ for $e=2$ and the set prediction $\frac{e-c}{2}-\frac{\sqrt{(e+c)^{2}-4}}{2} \leq i^{*} \leq \frac{e-c}{2}+\frac{\sqrt{(e+c)^{2}-4}}{2}$ for $e>2$.

Table 1 provides an overview of the optimality predictions based on expected utility maximization or optimal satisficing, i.e., optimal aspiration formation and choice making.

Table 1: Optimality predictions

\begin{tabular}{|c|c|c|c|c|}
\hline \multirow{2}{*}{\multicolumn{2}{|c|}{ cases }} & \multirow[t]{2}{*}{ optimal investment } & \multicolumn{2}{|r|}{ optimal satisficing } \\
\hline & & & in investing & in aspiration formation \\
\hline$c>0$ & $e \geq 2$ & $i^{*}=\frac{e-c}{2}-\frac{\sqrt{(e+c)^{2}-4}}{2}$ & $0 \leq i \leq i^{*}$ & $\bar{A}=\min \{1,(e-i)(c+i)\} ; \underline{A}=(e-i) c$ \\
\hline \multirow[t]{2}{*}{$c=0$} & $e=2$ & $i^{*}=1$ & $i^{*}=1$ & $\bar{A}=1 ; \underline{A}=0$ \\
\hline & $e>2$ & 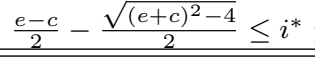 & $\leq i \frac{e-c}{2}+\frac{\sqrt{(e+c)^{2}-4}}{2}$ & $\bar{A}=1 ; \underline{A}=0$ \\
\hline
\end{tabular}

Notes: Optimality predictions for investing and aspiration formation based on expected utility maximization (left column) and optimal satisficing (middle, and right columns).

The set of satisficing choices and aspiration profiles in the sense of $\bar{A} \leq \bar{P}(i)$ and $\underline{A} \leq \underline{P}(i)$ becomes empty when aspirations are too ambitious, whereas when aspirations are moderate, the set is rather large. In view of previous experiences (Güth et al., 2009), aspiration formation was incentivized by paying for aspirations only when satisficing them; no payment was received otherwise. Thus, a participant with an aspiration profile $(\bar{A}, \underline{A})$ and choice $i$ earns $€ 14$ 
- in case (1), with probability $\bar{A}$ if $\bar{P}(i) \geq \bar{A}$ and zero probability otherwise

- in case (2), with probability $\underline{A}$ if $\underline{P}(i) \geq \underline{A}$ and zero probability otherwise

where 0 probability of earning $€ 14$ means earning $€ 4$ with probability 1 . Incentivizing satisficing in this seemingly cruel way (we later on refer to $\bar{A} \geq \bar{P}(i)$ or $\underline{A} \geq \underline{P}(i)$ as "economic suicide") arguably increases the cognitive load in the satisficing treatments with three payoff relevant choices, namely $i, \bar{A}$ and $\underline{A}$ instead of only one. But then neither optimality concept appeals to this cognitive load which it implies. But because of cognitive load we predict only satisficing but not its optimality.

Hypothesis 2 (Non-Optimal Satisficing): Participants learn to satisfice, but aspiration profiles $(\bar{A}, \underline{A})$ are, at least initially, non-optimal, i.e., one could increase either $\bar{A}$ or $\underline{A}$ without having to decrease the other. Furthermore, many $i$ choices will not be set optimal in the sense of optimally satisficing choices.

We predicted some "burning money" in the sense of small positive differences in $\bar{P}(i)-\bar{A}$ and $\underline{P}(i)-\underline{A}$. Compared to "burning money", we predicted significantly less evidence of "committing suicide" which occurs when $\bar{A}>\bar{P}(i)$ or $\underline{A}>\underline{P}(i)$. This means that the chance of earning 14 is lost when earning $\bar{A} \cdot \delta(\bar{P}(i) \geq \bar{A})$ or $\underline{A} \cdot \delta(\underline{P}(i) \geq \underline{A})$ in the boom and doom cases, respectively, with $\delta(\cdot)$ denoting the indicator function that takes the value 1 if its condition is satisfied and 0 otherwise.

When a probability $0<p<1$ is experimentally induced, a participant might use it for intrapersonal payoff aggregation or for aspiration formation and adjustment (see Sauermann and Selten, 1962). The latter could mean to be more ambitious in the more likely case (1) or (2), e.g., by increasing the respective aspiration level, $\bar{A}$ or $\underline{A}$.

Hypothesis 3 (Probability-Use Hypothesis): Participants will react mainly qualitatively to information about the probability $p$ when choosing or forming aspirations.

When participants are not informed of the probability $p$, they are partly asked to generate a subjective probability $\hat{p}=1 / 2$, e.g., $p=1 / 2$ due to the principle of insufficient reason. However, 
$\hat{p} \neq p$ does not change $i^{*}$ and does not call into question the optimality predictions in Table 3 . Experiences with and without experimentally induced prior probabilities, to the best of our knowledge, have found few differences in their choice data.

\section{Experimental protocols}

The four between subjects treatments implemented in the experiment (T1,T2, T3 and T4) rely on the $2 \times 2$ factorial design, choice format and probability information, presented in Table 2 . These treatments differ in whether probability $p$ is experimentally induced (T1 and T3) and on whether only the choice $\mathrm{i}(\mathrm{T} 1$ and $\mathrm{T} 2)$ or aspirations $(\bar{A}, \underline{A})$ and choice $i$ are elicited (T3 and T4).

Table 2: The $2 \times 2$-factorial between subjects treatments

\begin{tabular}{|c|c|c|}
\hline Choice Format & \multicolumn{2}{|c|}{ Probability Information } \\
\hline & $p$ given & $p$ unknown \\
\hline $\begin{array}{l}I \text {-treatment } \\
\text { (direct } i \text {-choice) }\end{array}$ & $\begin{array}{l}\mathrm{T} 1: i_{1}, i_{2}, i_{3}, i_{4}, i_{5}, i_{6} \\
\quad \longmapsto \text { final choice of } i\end{array}$ & $\begin{array}{c}\text { T2: first } \hat{p} \\
\text { then. } i_{1}, i_{2}, i_{3}, i_{4}, i_{5}, i_{6} \\
\longmapsto \text { final choice of } i\end{array}$ \\
\hline $\begin{array}{l}S \text {-treatment } \\
\text { (first aspiration profiles } \\
\text { then } i \text {-choice) }\end{array}$ & $\begin{array}{c}\text { T3: first. } \bar{A}, \underline{A} \\
\text { then } i_{1}, i_{2}, i_{3}, i_{4}, i_{5}, i_{6} \\
\longmapsto \text { final choice of } i\end{array}$ & $\begin{array}{c}\text { T4: first } \bar{A}, \underline{A} \\
\text { then } i_{1}, i_{2}, i_{3}, i_{4}, i_{5}, i_{6} \\
\longmapsto \text { final choice of } i\end{array}$ \\
\hline
\end{tabular}

For each treatment, we conducted three sessions with student participants recruited from Luiss University from different fields of study (mainly economics, law and political science) using Orsee software (Greiner, 2015). The number of participants per treatment varied from 71 to 78 . Overall, we employed a total of 302 participants. The experiment was fully computerized using Z-Tree (Fischbacher, 2007). Sessions lasted approximately 90 minutes. After each session, participants answered a brief questionnaire, mainly to collect demographic information, before being privately paid in cash for a randomly selected round. No one participated in more than one session. 


\subsection{Choice tasks}

At the beginning of each round each subject was endowed with an amount of money that could be allocated in two kinds of investment: a risk-free bond with constant repayment factor, independent of the market condition, and a risky asset whose repayment factor changed with the market condition and the amount invested in it. The market could be in good or bad conditions whose probabilities ( $p$ and $(1-p)$, respectively) were communicated in each round in $\mathrm{T} 1$ and $\mathrm{T} 3$ but were unknown in $\mathrm{T} 2$ and T4.

In all treatments, the allocation choices did not affect directly the potential earning. They only affected the probability or earning $€ 14$ instead of $€ 4$. In each round, before making the final decision on how much to invest in the risky asset $(i)$, subjects could try and visualize the effects of own choices on the probability of earning $€ 14$ in the two different market conditions by scrolling the cursor on the bar. The subjects had several attempts before the final one. They could move the cursor at most 6 times (the number of attempts, as well as the time elapsed in each round were clearly shown in the screenshots).

In T3 and T4, in each round before the investment choice, subjects were asked to state the probability of earning $€ 14$ instead of $€ 4$ that would make them satisfied, both for market in good and bad condition separately. These satisficing probability could be modified only once in each round. The comparison between their level and the probability of earning $€ 14$ or $€ 4$ implied by the investment choice determined the final potential earning, as it will be explained in 4.3.

\subsection{Parameters constellations}

The 18 cases in Table 3 were constructed by neglecting that $\bar{P}(i)$ cannot exceed 1 and imposing $P^{\circ}\left(i^{\circ}\right)=(1-p)\left(e-i^{\circ}\right) c+p\left(e-i^{\circ}\right)\left(c+i^{\circ}\right)=\left(e-i^{\circ}\right)\left(c+p i^{\circ}\right)=0.9$ for the unconstrained optimal choice $i^{\circ}$ across all cases. The actual optimal expected success probability, due to $\bar{P}(i) \leq 1$, varies considerably (see $P\left(i^{*}\right)$ in Table 3 ), namely, from 0.23 to 0.9 . The cases are defined by the endowment $e$ and the $i^{\circ} / e$-investment share of the unconstrained optimal investment $i^{\circ}$. Together, these determine the parameters $p$ and $c$ when imposing $P\left(i^{\circ}\right)=0.9$.

The different parameter constellations include the "Golden Mean", i.e. $i^{*}=e / 2$, with $c=0$ and 
$e=2$, and exclude $\underline{P}(i)=(e-i) c \geq 1$ via $e c<1$. Moreover, the parameter constellations capture some rather small and at least some rather large probabilities $p$ in the range $0<p<1$, which are payoff- but not optimal-choice relevant.

All participants confronted the parameter constellations twice in two successive phases, each with 18 rounds and in a random order.

Table 3: Cases and optimal payoffs

\begin{tabular}{l|ccc|ccc|ccc}
\hline \hline Case & $e$ & $c$ & $p$ & $i^{*}$ & $i^{\circ}$ & $q^{\circ}=\frac{i^{\circ}}{e}$ & $P\left(i^{*}\right)$ & $\bar{P}\left(i^{*}\right)$ & $\underline{P}\left(i^{*}\right)$ \\
\hline 1 & 2 & 0.00 & 0.90 & 1 & 1.00 & $6 / 12$ & 0.90 & 1 & 0.00 \\
2 & 2 & 0.22 & 0.66 & 0.41 & 0.83 & $5 / 12$ & 0.78 & 1 & 0.35 \\
3 & 2 & 0.34 & 0.51 & 0.22 & 0.67 & $4 / 12$ & 0.81 & 1 & 0.60 \\
4 & 2 & 0.40 & 0.40 & 0.14 & 0.50 & $3 / 12$ & 0.85 & 1 & 0.75 \\
5 & 2 & 0.43 & 0.32 & 0.09 & 0.33 & $2 / 12$ & 0.88 & 1 & 0.82 \\
6 & 2 & 0.45 & 0.27 & 0.07 & 0.17 & $1 / 12$ & 0.90 & 1 & 0.87 \\
7 & 3 & 0.00 & 0.40 & 0.38 & 1.50 & $6 / 12$ & 0.40 & 1 & 0.00 \\
8 & 3 & 0.15 & 0.29 & 0.21 & 1.25 & $5 / 12$ & 0.59 & 1 & 0.42 \\
9 & 3 & 0.23 & 0.23 & 0.12 & 1.00 & $4 / 12$ & 0.74 & 1 & 0.66 \\
10 & 3 & 0.27 & 0.18 & 0.07 & 0.75 & $3 / 12$ & 0.83 & 1 & 0.79 \\
11 & 3 & 0.29 & 0.14 & 0.05 & 0.50 & $2 / 12$ & 0.88 & 1 & 0.86 \\
12 & 3 & 0.30 & 0.12 & 0.04 & 0.25 & $1 / 12$ & 0.90 & 1 & 0.89 \\
13 & 4 & 0.00 & 0.23 & 0.27 & 2.00 & $6 / 12$ & 0.23 & 1 & 0.00 \\
14 & 4 & 0.11 & 0.17 & 0.15 & 1.67 & $5 / 12$ & 0.52 & 1 & 0.42 \\
15 & 4 & 0.17 & 0.13 & 0.09 & 1.33 & $4 / 12$ & 0.71 & 1 & 0.67 \\
16 & 4 & 0.20 & 0.10 & 0.05 & 1.00 & $3 / 12$ & 0.81 & 1 & 0.79 \\
17 & 4 & 0.22 & 0.08 & 0.03 & 0.67 & $2 / 12$ & 0.88 & 1 & 0.87 \\
18 & 4 & 0.22 & 0.07 & 0.03 & 0.33 & $1 / 12$ & 0.88 & 1 & 0.87 \\
\hline \hline
\end{tabular}

\subsection{Earnings}

A subject could earn either $€ 14$ or $€ 4$ in addition to the show-up fee of $€ 4$. Participants are paid for one random round selected by the computer at the end of the experiment.

In T1 and T2, earnings depended on the investment choice and the random event, whereas in T3 and T4, earnings depended on the investment choice, the random event and the aspiration levels $(\bar{A}, \underline{A}$ for winning $€ 14$ ). An aspiration level that did not exceed the probability of winning $€ 14$ (in either boom or doom cases) determined the probability of winning $€ 14$. If the aspiration level exceeded the probability of winning $€ 14$ for the $i$ choice (in either boom or doom), the probability of winning $€ 14$ was nil (but one received $€ 4$ with probability 1 ). For an example of the payoffs in T3 and T4, see Figure 10 in Appendix B: the boom aspiration level is $60 \%$, lower than the probability represented 
by the left bar. The doom aspiration level is higher than the overall probability represented by the right bar. Since $p=0.14$, doom results with probability 0.86 . As aspiration $\underline{A}$ exceeds the level $\underline{P}(i)=(e-i) c$, i.e., the chance of earning $€ 14$ in the case of doom is nil, and the probability of earning $€ 14$ is only $0.6 \times 0.14=0.084$.

\section{Assessing (Sub) Optimality For $c>0$}

We consider each choice as an observation to test optimality in $\mathrm{T} 1$ and $\mathrm{T} 2$ as postulated by expected utility maximization, and in T3 and T4 following optimal satisficing in investing and in forming aspiration. However, when assessing the extent of suboptimality and non-optimal satisficing, one might take into account which choices are made by the same participant and consider only the individual averages as independent. ${ }^{15}$

Although repeated slider use should quickly reveal that increasing $i$ in the interval from $i^{*}$ to $i^{\circ}$ is suboptimal, someone solving the unconstrained optimization task analytically might have overlooked this. We therefore checked (sub)optimality in view of $i^{\circ}$, although our main focus is, of course, the optimal choice $i^{*}$ and the optimal interval $0 \leq i \leq i^{*}$. Analyzing suboptimality across all 15 cases with $c>0$ via deviations $i^{\circ}-i$ across treatments T1, T2, T3, T4 and phases 1 and 2 (see Figure 11 in Appendix B) consistently reveals a positive mode, meaning that $i<i^{\circ}$ is more focal. This could point in the direction of optimality, $i^{*}$. In fact, (see Figure 3 ) the modes of the deviations $i-i^{*}$ from optimality are close to 0 for all treatments (the much longer tails in the range $i>i^{*}$ are due to $\frac{i^{*}}{e}$ usually being small in the unit interval).

In the action space, we compare actual investments with optimal ones aggregated across all 15 cases of either phase 1 or phase 2 , as well as for all $30 i$ choices with $c>0$ across treatments (see Figure 3). Some will be discussed in more detail via pairwise comparisons of treatments. What we observe so far is a tighter distribution around $i^{*}$ and stronger experience from phase 1 to 2 for T1 and $\mathrm{T} 2 .^{16}$

\footnotetext{
${ }^{15}$ We will often employ both possibilities by reporting significance levels based on each choice and individual averages, with the latter in brackets.

${ }^{16}$ Figure 11 in Appendix B shows the difference between the actual investment choice $(i)$ and the unconstrained optimal investment $(i \circ)$.
} 
Figure 3: Deviation $i^{*}-i$ by Phase and Treatment
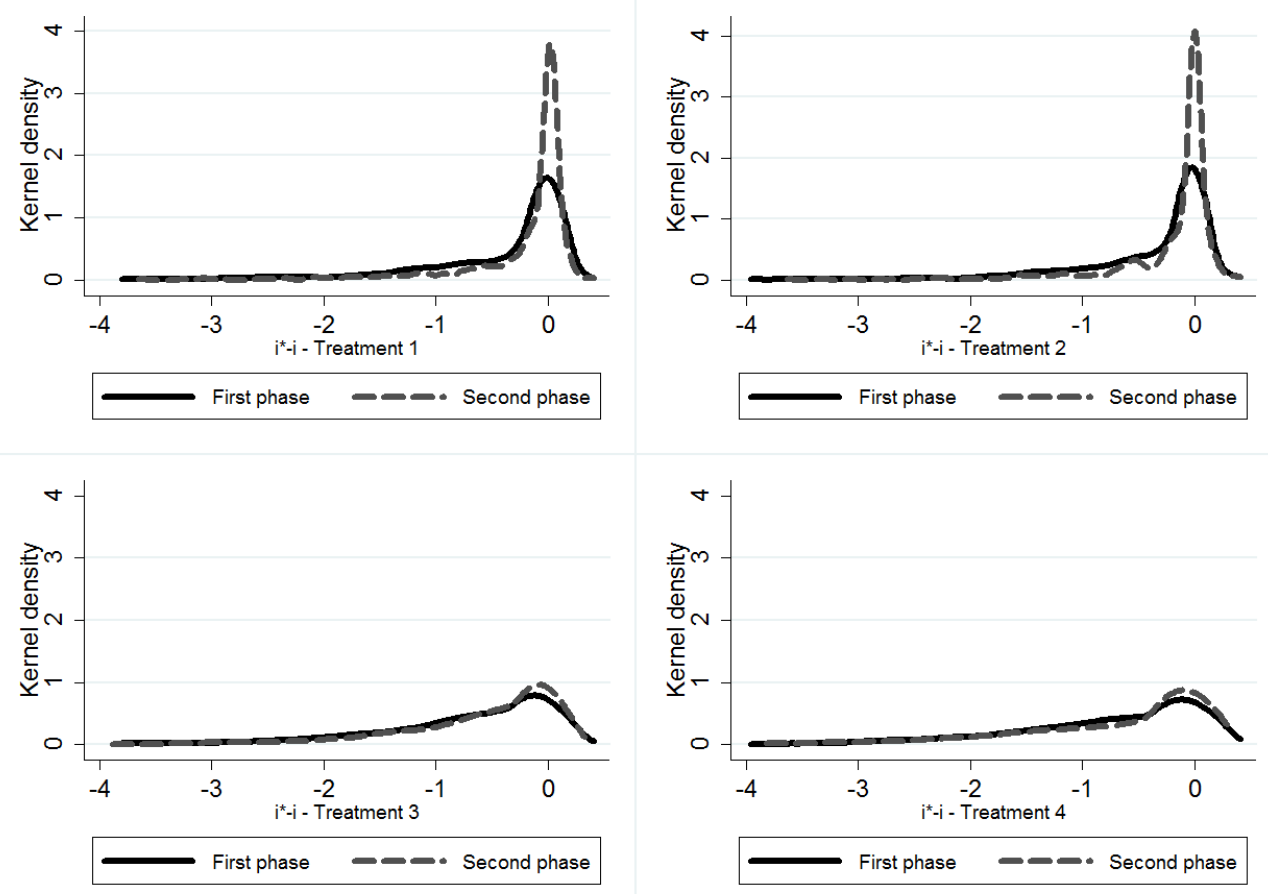

Notes: Kernel density function for cases with $c>0$.

Table 4 displays the average $i$ choices, as well as their frequencies and $\%$ shares for $i \leq i^{*}$, $i^{*}<i \leq i^{\circ}$ and $i>i^{\circ}$. The share of set-optimal $i$ choices $\left(i<i^{*}\right)$ is considerably larger in $\mathrm{T} 1$ and T2. Treatments T3 and T4 asking for aspirations $\bar{A}$ and $\underline{A}$ fare worse. Specifically, the share of $i$ choices with $i>i^{\circ}$ is much higher in T3 and T4 than in T1 and T2. It seems that incentivized aspiration formation crowds out rather than promotes better decision making. Note the low shares of $i$ choices in the interval $i \leq i^{*}$ even though the number of choices in this interval increases considerably from phase 1 to phase 2, especially in T1 and T2.

The influence of $q^{*}=i^{*} / e$ is demonstrated in Table 5, which distinguishes between cases with $q^{*} \leq 0.1$ and $0.1<q^{*} \leq 0.5$. For all four treatments, the share of $i$ choices with $i \leq i^{*}$ is always larger when $q^{*}$ exceeds 0.1 , i.e., the direction of deviation from the optimal choice $i^{*}$ is mainly determined by the lengths of the $q=i / e$ intervals to the left and right of $i^{*} / e$. However, 
Table 4: Average level $i$ by restricted and unrestricted optimal investment intervals

\begin{tabular}{ll|ccc|ccc|ccc}
\hline \hline & & \multicolumn{3}{|c}{$i \leq i^{*}$} & \multicolumn{3}{c}{$i^{*}<i \leq i^{\circ}$} & \multicolumn{3}{c}{$i>i^{\circ}$} \\
\hline & & $i$ & obs & $\%$ & $i$ & obs & $\%$ & $i$ & obs & $\%$ \\
\hline T1 & Phase 1 & 0.060 & 369 & 31.54 & 0.323 & 480 & 41.03 & 1.366 & 321 & 27.44 \\
& Phase 2 & 0.058 & 532 & 45.47 & 0.260 & 450 & 38.46 & 1.195 & 188 & 16.07 \\
\hline T2 & Phase 1 & 0.086 & 292 & 25.04 & 0.424 & 694 & 59.52 & 1.383 & 180 & 15.44 \\
& Phase 2 & 0.076 & 422 & 36.38 & 0.321 & 622 & 53.62 & 1.128 & 116 & 10.00 \\
\hline T3 & Phase 1 & 0.076 & 176 & 15.64 & 0.417 & 413 & 36.71 & 1.369 & 536 & 47.64 \\
& Phase 2 & 0.068 & 207 & 18.40 & 0.378 & 431 & 38.31 & 1.295 & 487 & 43.29 \\
\hline T4 & Phase 1 & 0.114 & 164 & 15.40 & 0.400 & 373 & 35.02 & 1.431 & 528 & 49.58 \\
& Phase 2 & 0.107 & 158 & 14.84 & 0.323 & 452 & 42.44 & 1.527 & 455 & 42.72 \\
\hline \hline
\end{tabular}

Notes: Average for 15 cases where $c>0$.

Table 5: Average level $i$ by restricted and unrestricted optimal investment intervals and by $q^{*}$ levels

\begin{tabular}{ll|cccc|cccc}
\hline \hline & \multicolumn{9}{|c}{$q$ Low } \\
& & $\mathrm{T} 1$ & $\mathrm{~T} 2$ & $\mathrm{~T} 3$ & $\mathrm{~T} 4$ & $\mathrm{~T} 1$ & $\mathrm{~T} 2$ & $\mathrm{~T} 3$ & $\mathrm{~T} 4$ \\
\hline$i \leq i^{*}$ & $i$-mean & 0.034 & 0.055 & 0.042 & 0.064 & 0.193 & 0.171 & 0.201 & 0.203 \\
& $\%$ & 37.43 & 27.69 & 15.95 & 11.59 & 45.51 & 50.16 & 24.00 & 38.03 \\
$i^{*}<i \leq i^{\circ}$ & $i$-mean & 0.272 & 0.372 & 0.386 & 0.341 & 0.472 & 0.421 & 0.488 & 0.482 \\
& $\%$ & 41.22 & 60.69 & 38.56 & 39.54 & 30.13 & 29.90 & 30.67 & 33.45 \\
$i>i^{\circ}$ & $i$-mean & 1.329 & 1.388 & 1.356 & 1.505 & 1.154 & 0.884 & 1.189 & 1.150 \\
& $\%$ & 21.35 & 11.61 & 45.94 & 48.86 & 24.36 & 19.94 & 45.33 & 28.52 \\
& Obs. & 2028 & 2015 & 1950 & 1846 & 312 & 311 & 300 & 284 \\
\hline \hline
\end{tabular}

Notes: Average results for the 15 cases with $c>0$ when $q^{*} \leq 0.10$ is Low, and $0.10<q^{*} \leq 0.5$ is High.

these shares are considerably smaller for T3 and T4 than for T1 and T2, irrespective whether $q^{*}$ is "Low"or "High".

In the payoff space, we compare the following:

- expected payoff losses

$$
P\left(i^{*}\right)-P(i)=(1-p)\left(e-i^{*}\right) c+p-(1-p)(e-i) c-p \cdot \min \{1,(e-i)(c+i)\}
$$

separately for phase 1 and 2, as well as for 30 treatments (see Figure 4) and

- separate payoff losses for boom (see Figure 12 in Appendix B) and doom (see Figure 13 in Appendix B) in order to determine whether suboptimality is due to special concerns in either boom or doom cases.

The payoff distributions are consistent with the $i$ choice distributions, in particular, deviations are closer to zero in the payoff space for $\mathrm{T} 1$ and $\mathrm{T} 2$ and more so in phase 2 in all three dimensions, 
Figure 4: Distance in payoff space: $P\left(i^{*}\right)-P(i)$ by phase
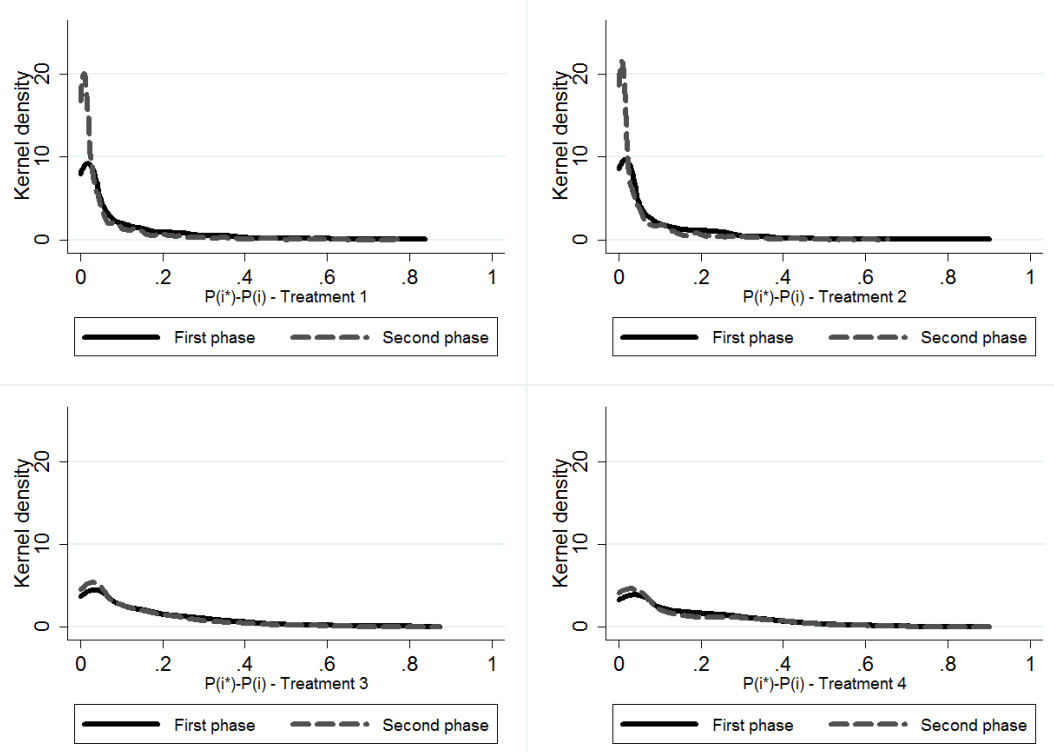

Notes: Kernel density function, considering only cases where $c>0$; Treatment $2 P\left(i^{*}\right)$ has been computed based on objective probabilities $p$.

$P\left(i^{*}\right)-P(i), \bar{P}\left(i^{*}\right)-\bar{P}(i)$ and $\underline{P}\left(i^{*}\right)-\underline{P}(i)$.

We now consider pairwise treatment comparisons based on data for 15 cases with $c>0$. Pairwise treatment comparisons allow us to control specifically for whether $p$ information is granted and which additional data are elicited.

(a) T1 $\leftrightarrow \mathrm{T} 2$ : differ in that $p$ is known in T1 but not in T2, which also asks for $\hat{p}$, the subjectively stated probability of a boom.

Figure 5, displaying the densities of $p$ and $\hat{p}$ for all participants of T2 separately for both phases, reveals a dominance of wishful thinking in both phases and hardly any learning. ${ }^{17}$ Such optimism, however, does not account for the suboptimal choices, since $i^{*}$ does not depend on $p$ or $\hat{p}$. When we test for differences in probabilities between T1 and T2 (see Table 14 in Appendix B) the excessive optimism is significant $(p<0.00)$, which is insignificantly $(p>0.05)$ reduced by experience.

\footnotetext{
${ }^{17}$ In each round of T2, we allow participants to modify the stated probability once: this actually occurred $3 \%$ and $2 \%$ of the times in phase 1 and 2 , respectively.
} 
Figure 5: Comparison of $p$ and $\hat{p}$ for $\mathrm{T} 2$
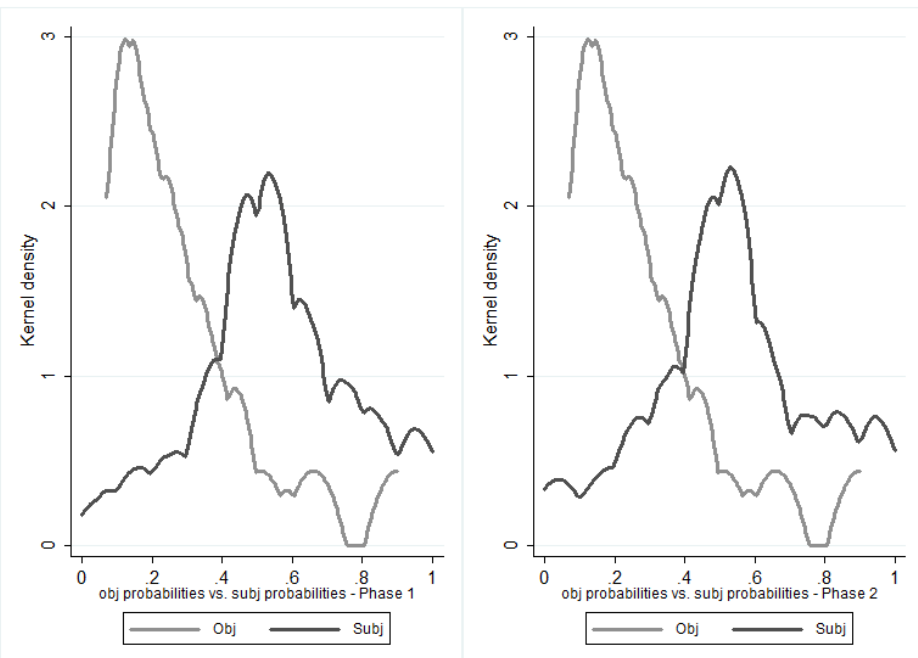

Table 6: Action and payoff space comparison - T1 vs. T2

\begin{tabular}{|c|c|c|c|c|c|c|c|c|c|c|c|c|}
\hline & \multicolumn{3}{|c|}{$i^{*}-i$} & \multicolumn{3}{|c|}{$P\left(i^{*}\right)-P(i)$} & \multicolumn{3}{|c|}{$\bar{P}\left(i^{*}\right)-\bar{P}(i)$} & \multicolumn{3}{|c|}{$\underline{P}\left(i^{*}\right)-\underline{P}(i)$} \\
\hline T1 vs. T2 & T1 & $\mathrm{T} 2$ & WRST(Ind.) & $\mathrm{T} 1$ & T2 & WRST & T1 & T2 & WRST & $\mathrm{T} 1$ & $\mathrm{~T} 2$ & WRST \\
\hline All sample & -0.305 & -0.282 & $0.013(0.004)$ & 0.076 & 0.070 & 0.056 & 0.063 & 0.043 & 0.000 & 0.075 & 0.069 & 0.049 \\
\hline Phase 1 & -0.409 & -0.369 & $0.427(0.409)$ & 0.095 & 0.087 & 0.315 & 0.056 & 0.040 & 0.001 & 0.102 & 0.091 & 0.666 \\
\hline Phase 2 & -0.201 & -0.195 & $0.005(0.029)$ & 0.057 & 0.054 & 0.063 & 0.071 & 0.045 & 0.000 & 0.049 & 0.046 & 0.015 \\
\hline KST(Ind.) & $0.000(0.000)$ & $0.000(0.000)$ & & 0.000 & 0.000 & & 0.000 & 0.000 & & 0.000 & 0.000 & \\
\hline
\end{tabular}

Notes: A Wilcoxon Rank Sum Test (WRST) for two independent samples is used to compare across treatments, and a KolmogorovSmirnov equality of distributions test (KST) for the analysis across phases. The parentheses report the tests on individual averages. For these tests, p-values are reported. In T2, $P\left(i^{*}\right)$ is computed based on objective probabilities $p$.

Table 6 reveals a larger average deviation $i^{*}-i$ for T1 than for T2, which seems mainly due to (significant) differences in behavior in phase 2. In the payoff dimensions $P(i), \bar{P}(i)$ and $\underline{P}(i)$, the differences are significantly affected by phase with only $\bar{P}\left(i^{*}\right)-\bar{P}(i)$ increasing from phase 1 to phase 2 .

(b) T1 $\leftrightarrow$ T3: Participants in both treatments are aware of probability $p$ and differ only in that participants in $\mathrm{T} 3$ also have to form aspirations, which they can revise only once.

In Table 7, we restrict the comparison to aspects for which both treatments provide data. In spite of what the overall comparisons across all treatments suggest, homogeneity of deviation from 
Table 7: Action and payoff space comparison - T1 vs. T3

\begin{tabular}{|c|c|c|c|c|c|c|c|c|c|c|c|c|}
\hline & \multicolumn{3}{|c|}{$i^{*}-i$} & \multicolumn{3}{|c|}{$P\left(i^{*}\right)-P(i)$} & \multicolumn{3}{|c|}{$\overline{\bar{P}}\left(i^{*}\right)-\bar{P}(i)$} & \multicolumn{3}{|c|}{$\underline{\underline{P}}\left(i^{*}\right)-\underline{P}(i)$} \\
\hline T1 vs. T3 & T1 & T3 & WRST(Ind.) & T1 & T3 & WRST & T1 & T3 & WRST & T1 & T3 & WRST \\
\hline All sample & -0.305 & -0.650 & $0.000(0.000)$ & 0.076 & 0.139 & 0.000 & 0.063 & 0.036 & 0.000 & 0.075 & 0.165 & 0.000 \\
\hline Phase 1 & -0.409 & -0.699 & $0.000(0.000)$ & 0.087 & 0.150 & 0.000 & 0.056 & 0.036 & 0.000 & 0.102 & 0.177 & 0.000 \\
\hline Phase 2 & -0.201 & -0.600 & $0.000(0.000)$ & 0.054 & 0.129 & 0.000 & 0.071 & 0.036 & 0.000 & 0.049 & 0.152 & 0.000 \\
\hline KST(ind.) & $0.000(0.000)$ & $0.001(0.000)$ & & 0.000 & 0.010 & & 0.000 & 0.917 & & 0.000 & 0.005 & \\
\hline
\end{tabular}

Notes: A Wilcoxon Rank Sum Test (WRST) for two independent samples is used to compare across treatments, and a KolmogorovSmirnov equality of distributions test (KST) for the analysis across phases. The parentheses report the tests on individual averages. For these tests, p-values are reported.

optimality in the action and payoff space, as well as across phases, is not rejected, except for the smaller payoff losses $\bar{P}\left(i^{*}\right)-\bar{P}(i)$ in phase 2 of T3.

(c) T2 $\leftrightarrow$ T3: Both treatments burden participants by eliciting additional choices, $\hat{p}$ in the case of $\mathrm{T} 2$ and $\bar{A}, \underline{A}$ in the case of $\mathrm{T} 3$, where of course, the latter seems to be more cognitively demanding.

Table 8: Action and payoff space comparison - T2 vs. T3

\begin{tabular}{l|ccc|cccc|ccc|cc}
\hline \hline & \multicolumn{4}{|c}{$i^{*}-i$} & \multicolumn{4}{c}{$P\left(i^{*}\right)-P(i)$} & $\bar{P}\left(i^{*}\right)-\bar{P}(i)$ & \multicolumn{4}{c}{$\left(i^{*}\right)-\underline{P}(i)$} \\
\hline T2 vs. T3 & T2 & T3 & WRST(Ind.) & T2 & T3 & WRST & T2 & T3 & WRST & T2 & T3 & WRST \\
\hline All sample & -0.282 & -0.650 & $0.000(0.000)$ & 0.070 & 0.139 & 0.000 & 0.043 & 0.036 & 0.000 & 0.069 & 0.165 & 0.000 \\
Phase 1 & -0.369 & -0.699 & $0.000(0.000)$ & 0.087 & 0.150 & 0.000 & 0.040 & 0.036 & 0.000 & 0.091 & 0.177 & 0.000 \\
Phase 2 & -0.195 & -0.600 & $0.000(0.000)$ & 0.054 & 0.129 & 0.000 & 0.045 & 0.036 & 0.000 & 0.046 & 0.152 & 0.000 \\
KST(ind.) & $0.000(0.000)$ & $0.001(0.000)$ & & 0.000 & 0.010 & & 0.000 & 0.917 & & 0.000 & 0.005 \\
\hline \hline
\end{tabular}

Notes: A Wilcoxon Rank Sum Test (WRST) for two independent samples is used to compare across treatments, and a KolmogorovSmirnov equality of distributions test (KST) for the analysis across phases. The parentheses report the tests on individual averages. For these tests, p-values are reported. In T2, $P\left(i^{*}\right)$ is computed from objective probabilities $p$.

Again restricting the comparison to aspects for which both treatments provide data (see Table 8), one qualitative conclusion is that knowing $p$, only in T3, apparently does not help: average choice behavior and outcomes are closer to optimality in T2, although $\bar{P}\left(i^{*}\right)-\bar{P}(i)$ is smaller for T3 than for T2.

$(d) \mathrm{T} 1 \leftrightarrow \mathrm{T} 4$ and $\mathrm{T} 2 \leftrightarrow \mathrm{T} 4$

All statements for $\mathrm{T} 2 \leftrightarrow \mathrm{T} 3$ apply analogously (see Tables 16 and 15 in Appendix B). 


\section{Comparing Satisficing across Treatments}

Let us first compare how often aspirations were adjusted: in T3, aspirations were adjusted 25\% (35\%) of the time in phase 1 (phase 2), while in T4, aspirations were adjusted 35\% (39\%) of the time in phase 1 (phase 2). With experience, one engages in more frequent aspiration adaptation (Sauermann and Selten, 1962).

Table 9: Action and payoff space comparison - T3 vs. T4

\begin{tabular}{|c|c|c|c|c|c|c|c|c|c|c|c|c|}
\hline & \multicolumn{3}{|c|}{$\overline{i^{*}-i}$} & \multicolumn{3}{|c|}{$\overline{P P\left(i^{*}\right)-P(i)}$} & \multicolumn{3}{|c|}{$\overline{\bar{P}\left(i^{*}\right)-\overline{\bar{P}}(i)}$} & \multicolumn{3}{|c|}{$\underline{P}\left(i^{*}\right)-\underline{P}(i)$} \\
\hline T3 vs. T4 & T3 & $\mathrm{T} 4$ & WRST(Ind.) & T3 & $\mathrm{T} 4$ & WRST & T3 & $\mathrm{T} 4$ & WRST & T3 & $\mathrm{T} 4$ & WRST \\
\hline All sample & -0.650 & -0.718 & $0.114(0.000)$ & 0.139 & 0.152 & 0.197 & 0.036 & 0.030 & 0.015 & 0.165 & 0.175 & 0.275 \\
\hline Phase 1 & -0.699 & -0.749 & $0.348(0.003)$ & 0.150 & 0.161 & 0.131 & 0.036 & 0.035 & 0.613 & 0.177 & 0.185 & 0.488 \\
\hline Phase 2 & -0.600 & -0.688 & $0.207(0.041)$ & 0.129 & 0.142 & 0.820 & 0.036 & 0.025 & 0.003 & 0.152 & 0.165 & 0.391 \\
\hline KST(Ind.) & $0.001(0.000)$ & $0.000(0.000)$ & & 0.010 & 0.000 & & 0.917 & 0.842 & & 0.005 & 0.000 & \\
\hline
\end{tabular}

Notes: A Wilcoxon Rank Sum Test (WRST) for two independent samples is used to compare across treatments, and a KolmogorovSmirnov equality of distributions test (KST) for the analysis across phases. The parentheses report the tests on individual averages. For these tests, p-values are reported.

Regarding deviations from $i^{*}$ in the action and payoff space, Table 9 reveals a significant phase effect for $i^{*}-i, P\left(i^{*}\right)-P(i)$ and $\underline{P}\left(i^{*}\right)-\underline{P}(i)$ for both treatments and rejects homogeneity of payoff deviations $\bar{P}\left(i^{*}\right)-\bar{P}(i)$ across treatments for phase 2 .

Table 10: Share and average level of satisficing

\begin{tabular}{l|cc|cc}
\hline \hline & \multicolumn{3}{|c}{ T3 } & \multicolumn{2}{c}{ T4 } \\
& Phase 1 & Phase 2 & Phase 1 & Phase 2 \\
\hline \% of $(\bar{P}(i)-\bar{A} \geq 0) \&(\underline{P}(i)-\underline{A} \geq 0)$ & 77 & 81 & 80 & 83 \\
Obs & 1125 & 1125 & 1065 & 1065 \\
\hline$\%$ of $(\bar{P}(i)-\bar{A} \geq 0)$ & 97 & 98 & 97 & 98 \\
$\phi(\bar{P}(i)-\bar{A} \mid \bar{P}(i)-\bar{A} \geq 0)$ & 0.307 & 0.304 & 0.295 & 0.245 \\
s.d. & 0.15 & 0.18 & 0.17 & 0.19 \\
\hline \% of $(\underline{P}(i)-\underline{A} \geq 0)$ & 77 & 82 & 80 & 84 \\
$\phi(\underline{P}(i)-\underline{A} \mid \underline{P}(i)-\underline{A} \geq 0)$ & 0.212 & 0.230 & 0.165 & 0.149 \\
s.d. & 0.19 & 0.21 & 0.16 & 0.15 \\
\hline \hline
\end{tabular}

Notes: Average for 15 cases where $c>0$.

Tables 10 and 11 both rely on the finally confirmed $\bar{A}, \underline{A}$ aspiration profiles. Table 10 lists the percentage share of satisficing (top row) as combined percentage shares of $\bar{P}(i) \geq \bar{A}$ and $\underline{P}(i) \geq \underline{A}$. 
Table 11: Share and average level when $\bar{P}(i)<1$ and $\bar{P}(i)=1$

\begin{tabular}{l|cccc|cccc}
\hline \hline & \multicolumn{4}{|c}{$\bar{P}(i)<1$} & \multicolumn{4}{c}{$\bar{P}(i)=1$} \\
& \multicolumn{2}{|c}{ T3 } & \multicolumn{2}{c}{ T4 } & \multicolumn{2}{c}{ T4 } \\
& Ph. 1 & Ph. 2 & Ph. 1 & Ph. 2 & Ph. 1 & Ph. 2 & Ph. 1 & Ph. 2 \\
\hline Obs. & 216 & 242 & 198 & 181 & 909 & 883 & 867 & 884 \\
\% of $(\bar{P}(i)-\bar{A} \geq 0)$ & 86 & 89 & 86 & 90 & 100 & 100 & 100 & 100 \\
$\phi(\bar{P}(i)-\bar{A} \mid \bar{P}(i)-\bar{A} \geq 0)$ & 0.201 & 0.234 & 0.210 & 0.161 & 0.329 & 0.321 & 0.311 & 0.261 \\
s.d. & 0.15 & 0.20 & 0.16 & 0.15 & 0.14 & 0.17 & 0.16 & 0.19 \\
\hline \hline
\end{tabular}

Notes: We consider the 15 cases where $c>0$. Obs. refers to the total number of choices, where $\bar{P}(i)<1$ and $\bar{P}(i)=1$, and the share accounts for those consistent with condition $\bar{P}(i)-\bar{A}>0$.

Satisficing is prevalent in approximately $80 \%$ of all individual choices and increases slightly with experience. When we decompose the analysis between actual success and aspiration for boom and doom separately (Table 10, central and bottom rows) failures are mainly caused by not meeting the doom condition $\underline{P} \geq \underline{A}$, probably due to $\underline{P}(i)<1$ rendering it more difficult to specify a numerical visual slider cue.

To justify that failing to satisfice is due to numerically translating column heights in numerical aspirations when they are below 1 we compare the the cases $\bar{P}(i)<1$ and $\bar{P}(i)=1$. Table 11 separates the relatively few $i$ observations yielding $\bar{P}(i)<1$, where the same difficulty of $\underline{P}(i)<1$ arises. We conclude that, when $\bar{P}(i)<1$, the share of satisficing is higher than the doom cases where $\underline{P}(i)<1$ (see $\underline{P}(i)-\underline{A} \geq 0$ in Table 10).

The average distance, $\phi(\bar{P}(i)-\bar{A} \mid \bar{P}(i)-\bar{A} \geq 0)$, in Table 11 is consistently larger for $\bar{P}(i)=1$ than for $\bar{P}(i)<1$ : why guaranteeing $\bar{P}(i)=1$, as predicted by optimality, but not aspiring it? An interpretation could be that one aspires to a satisficingly high probability of earning more $(€ 14)$, suggesting that $\bar{P}(i)-\bar{A}$ is equally large for $\bar{P}(i)<1$ and $\bar{P}(i)=1$. The fact that $\bar{P}(i)-\bar{A}$ ranges from 0.161 to 0.234 for $\bar{P}(i)<1$ and from 0.261 to 0.329 for $\bar{P}(i)=1$ supports this explanation.

The scatter plots displaying the average individual $(\bar{P}-\bar{A}, \underline{P}-\underline{A})$ differences in T3 and T4 in Figure 6 suggest a positive correlation between these dimensions, specifically $0.27(0.42)$ for phase 1 (phase 2) in T3 and 0.50 (0.44) for phase 1 (phase 2) in T4, which are all statistically significant.

Setting $\bar{A}<\bar{P}(i)$ or $\underline{A}<\underline{P}(i)$ means sacrificing the probability of earning $€ 14$ to earn only $€ 4$, 
Figure 6: Scatter plots of individual average deviations $\bar{P}(i)-\bar{A}$ and $\underline{P}(i)-\underline{A}$ for T3 and T4
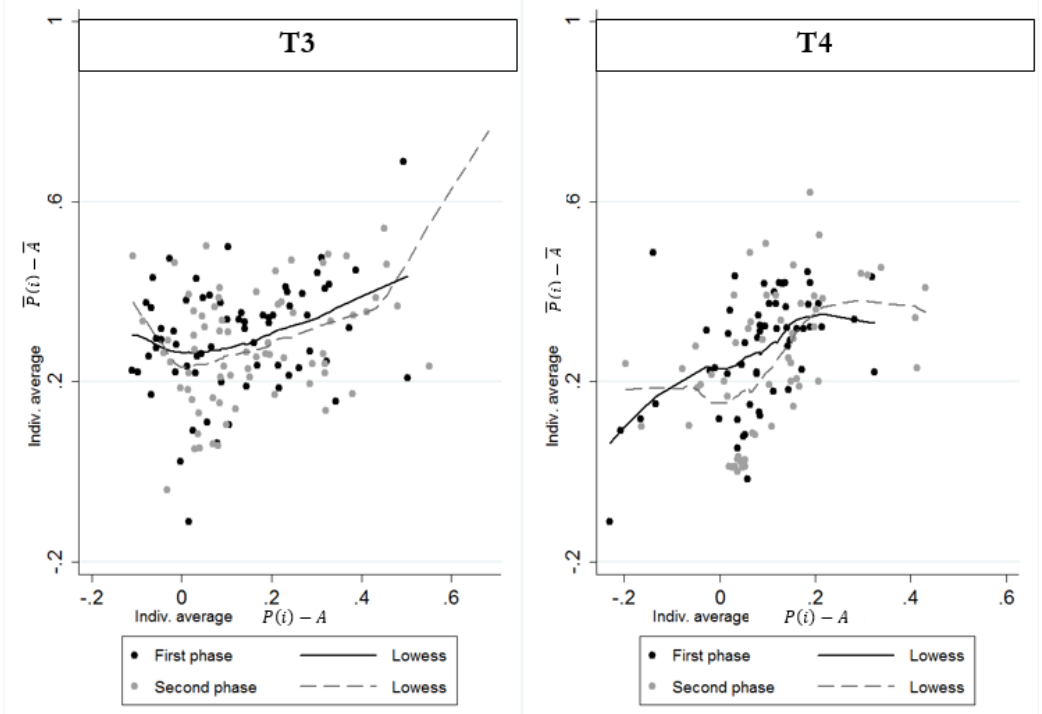

Notes: Individual average deviation for cases where $c>0$.

which is referred to as "burning money", $\overline{B M}=\max \{\bar{P}(i)-\bar{A}, 0\}$ and $\underline{B M}=\max \{\underline{P}(i)-\underline{A}, 0\}$. In our view, burning moderate amounts reveals a general skepticism of or timidity to exploit the investment choice $i$. Participants seemingly do not mind sacrificing some probability of earning more in order to compensate for slight misunderstandings or mistakes (see Güth et al., 2009, for similar results). However, as Figure 6 reveals, "burning money" may be more than a moderate sacrifice.

Whereas "burning money" does not question satisficing, "committing suicide" via $\bar{A}>\bar{P}(i)$ in case of $\bar{P}(i)<1, \overline{C S}$, or $\underline{A}>\underline{P}(i)$, committing suicide $\underline{C S}$, does. Figure 7 presents the evidence of "burning money" and "committing suicide" for all data (both phases of both treatments), as well the consequences for the chances to earn $€ 14$, where the left (right) diagram depicts boom (doom). When the aspiration exceeds what the $i$ choice yields, the chances to earn $€ 14$ are nil, and when "burning money", the aspired probability becomes the actual chance of earning $€ 14$. Figure 7 clearly reveals the expected and striking preponderance of "burning money" (this is confirmed by the kernel density plots in Figure 14 in Appendix B separately for boom (upper plots) and doom (lower plots) as well as for T3 (left plots) and T4 (right plots). The modes are in the $B M$ ranges with the 
Figure 7: Chances to earn $€ 14$ depending on aspirations
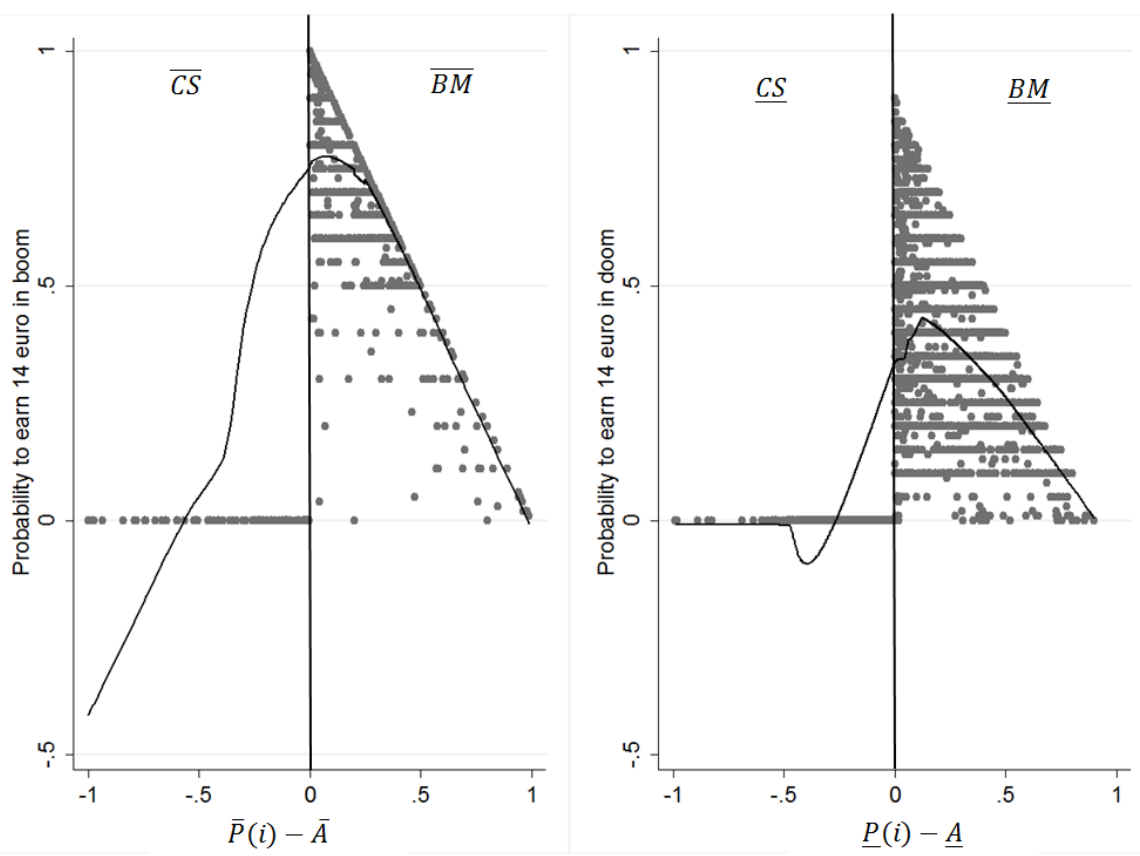

Notes: Pooled observations for phase 1, phase 2, Treatment 3 and Treatment 4 . The probability of winning $€ 14$ is the probability $\bar{A}$ (left graph) and $\underline{A}$ (right graph): these probabilities are positive when "burning money", $\overline{B M}$ respectively $\underline{B M}$, and the probability falls to 0 for "committing suicide", $\overline{C S}$ respectively $\underline{C S}$.

only remarkable reduction from phase 1 to phase 2 in case of $\overline{B M}$ in T4).

The top row of Table 12 shows that optimal aspiration formation is very unlikely $(\bar{A}=\bar{P}(i)$ is always below $5 \%$, and $\underline{A}=\underline{P}(i)$ is granted only twice) but more frequent in T4, as if information about $p$ crowds out better decision making. Table 12 also displays the expectedly high percentage shares of $B M$ and low $C S$ shares, with $\underline{C S}$ being more frequent than $\overline{C S}$.

$\mathrm{BM}$ and CS might be driven by neglegible errors, so we compare $B M$ and $C S$ across treatments and phases by tolerating $\epsilon$ amounts of "burning money" and "committing suicide" when smaller than $\epsilon=0.025$ (upper part of Table17 in Appendix B) and $\epsilon=0$ (lower part). Compared to the average amounts of "burning money" ( $\phi \overline{B M}, \phi \underline{B M})$ and "committing suicide" ( $\phi \overline{C S}, \phi \underline{C S})$ for $\epsilon=0$, the corresponding averages for $\epsilon=0.025$ are considerably larger: many deviations from optimal aspiration formation for given $i$ choices are beyond small margins. The striking reduction from phase 1 to phase 2 is restricted to "committing suicide" (see Table 17 in Appendix B). 
Table 12: "Burning Money" and "Committing Suicide" if $c>0$

\begin{tabular}{|c|c|c|c|c|c|c|}
\hline & \multicolumn{3}{|c|}{ T3 } & \multicolumn{3}{|c|}{ T4 } \\
\hline & Phase 1 & \multicolumn{2}{|l|}{ Phase 2} & Phase 1 & \multicolumn{2}{|l|}{ Phase 2} \\
\hline$\% \bar{P}(i)=\bar{A}$ & 0.31 & \multicolumn{2}{|l|}{0.67} & 2.35 & \multicolumn{2}{|l|}{4.98} \\
\hline Observations & 7 & \multicolumn{2}{|l|}{15} & 50 & \multicolumn{2}{|l|}{106} \\
\hline$\% \underline{P}(i)=\underline{A}$ & 0.00 & \multicolumn{2}{|l|}{0.09} & 0.00 & \multicolumn{2}{|l|}{0.00} \\
\hline Observations & 0 & 2 & & 0 & 0 & \\
\hline \multicolumn{7}{|c|}{ Burning Money } \\
\hline & Phase 1 & Phase 2 & KST & $\begin{array}{l}\text { Phase } 1 \\
4638\end{array}$ & $\begin{array}{l}\text { Phase } 2 \\
4418\end{array}$ & KST \\
\hline $\begin{array}{l}\% \frac{B M}{B(\overline{B M})} \\
\phi(\bar{m}\end{array}$ & $\begin{array}{l}48.31 \\
0.309\end{array}$ & $\begin{array}{l}48.18 \\
0.308\end{array}$ & 0.003 & $\begin{array}{l}40.58 \\
0.310\end{array}$ & 0.273 & 0.000 \\
\hline s.d. & 0.146 & 0.176 & & 0.157 & 0.177 & \\
\hline Observation & 1087 & 1084 & & 988 & 941 & \\
\hline$\% B M$ & 38.71 & 40.98 & & 40.14 & 41.97 & \\
\hline$\phi(\overline{(\underline{B M})})$ & 0.212 & 0.231 & 0.271 & 0.165 & 0.149 & 0.070 \\
\hline & 0.190 & 0.206 & & 0.160 & 0.150 & \\
\hline Observation & 871 & 922 & & 855 & 894 & \\
\hline \multicolumn{7}{|c|}{ Committing Suicide } \\
\hline & Phase 1 & Phase 2 & KST & Phase 1 & Phase 2 & KST \\
\hline$\% \overline{C S}$ & 1.38 & 1.16 & & 1.27 & 0.85 & \\
\hline$\phi(\overline{C S})$ & 0.351 & 0.212 & 0.020 & 0.369 & 0.152 & 0.031 \\
\hline s.d. & 0.303 & 0.206 & & 0.324 & 0.166 & \\
\hline Observation & 31 & 26 & & 27 & 18 & \\
\hline$\% \underline{C S}$ & 11.29 & 8.93 & & 9.86 & 8.03 & \\
\hline$\phi \overline{(\overline{C S})}$ & 0.172 & 0.139 & 0.064 & 0.202 & 0.173 & 0.275 \\
\hline & 0.150 & 0.130 & & 0.171 & 0.139 & \\
\hline Observation & 254 & 201 & & 210 & 171 & \\
\hline
\end{tabular}

Notes: The analysis refers to the p-values of Kolmogorov-Smirnov equality of distributions tests between phases. Cases with $c=0$ are excluded.

\section{The $c=0$ cases}

The $c=0$ cases are important, since they allow us to compare the point and set-valued optimality predictions differently.

- for $e=2$, even generically set-valued optimal satisficing becomes a point prediction coinciding with expected utility maximization;

- for $e>2$, even generically unique expected utility maximization becomes set-valued, again coinciding with optimal satisficing; and

- for $e=3$ and $e=4$, one can determine how the size of the optimal set affects the degree of (non)optimal behavior.

Figure 8 and Table 13 report the choice behavior for $c=0$. Figure 8 distinguishes between the different levels of endowment ( $e$ equal to 2, 3 and 4) and lists the percentage shares of $i$ choices below, equal to and larger than the optimal choices. Optimality is rare when point valued $(e=2)$ but predominant when set valued $(e>2)$. Surprisingly, optimality is less strongly supported in treatment $\mathrm{T} 1$ when its set prediction is larger $(e=4$ compared to $e=3)$. Only for treatments T3 


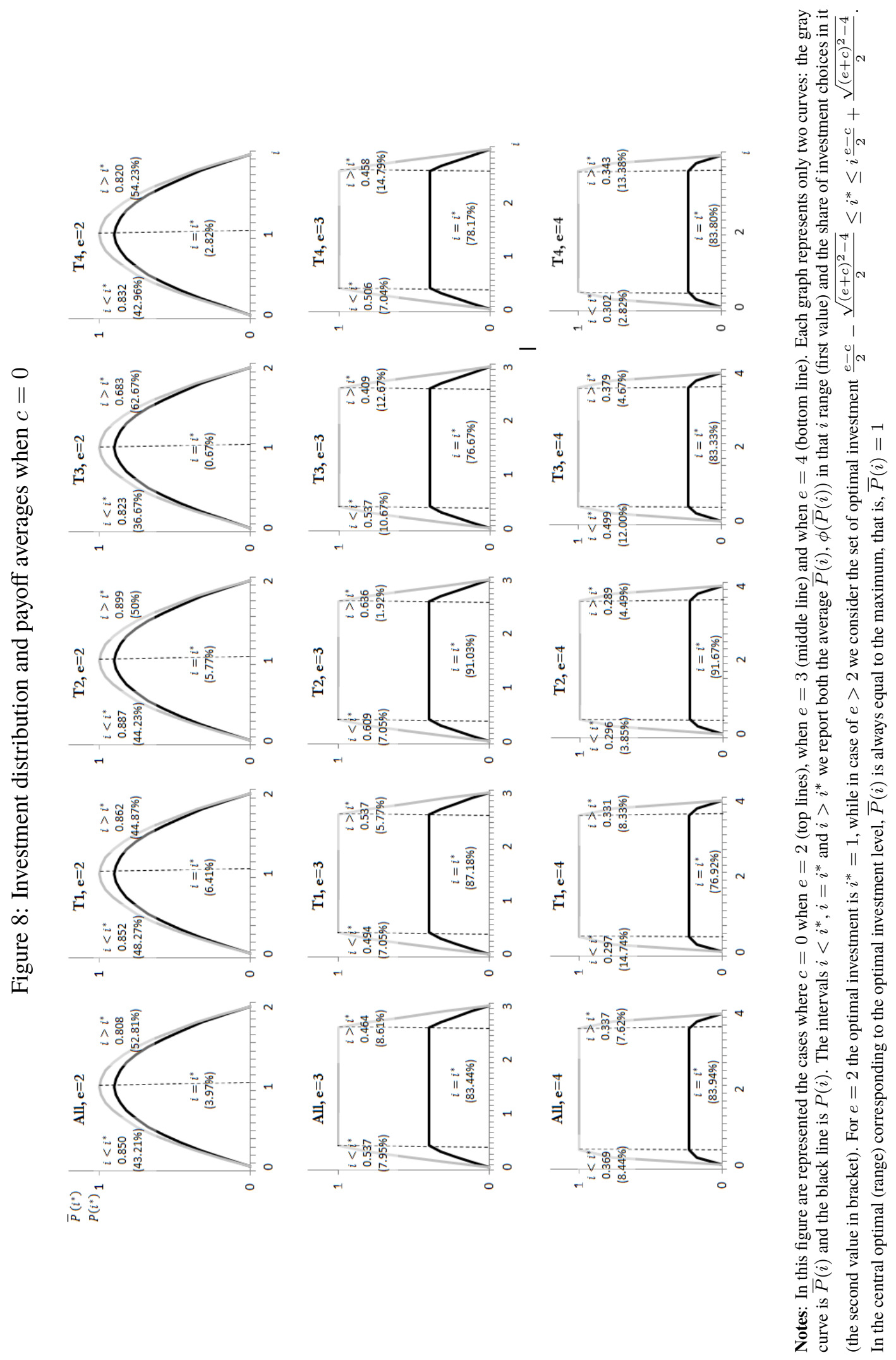


Table 13: Share and average level of satisficing when $c=0$

\begin{tabular}{l|cc|cc}
\hline \hline & \multicolumn{3}{|c}{ T3 } & \multicolumn{2}{c}{ T4 } \\
& Phase 1 & Phase 2 & Phase 1 & Phase 2 \\
\hline \% of $(\bar{P}(i)-\bar{A} \geq 0)$ & 84 & 88 & 89 & 88 \\
$\phi(\bar{P}(i)-\bar{A} \mid \bar{P}(i)-\bar{A} \geq 0)$ & 0.279 & 0.290 & 0.267 & 0.228 \\
s.d. & 0.17 & 0.21 & 0.17 & 0.20 \\
\hline & \multicolumn{4}{|c}{$\bar{P}(i)<1$} \\
\hline$\%$ of $(\bar{P}(i)-\bar{A} \geq 0)$ & 65 & 74 & 75 & 74 \\
$\phi(\bar{P}(i)-\bar{A} \mid \bar{P}(i)-\bar{A} \geq 0)$ & 0.217 & 0.214 & 0.229 & 0.201 \\
s.d. & 0.16 & 0.20 & 0.17 & 0.18 \\
Obs. & 105 & 105 & 97 & 99 \\
\hline & \multicolumn{4}{|c}{$\bar{P}(i)=1$} \\
\hline \% of $(\bar{P}(i)-\bar{A} \geq 0)$ & 100 & 100 & 100 & 100 \\
$\phi(\bar{P}(i)-\bar{A} \mid \bar{P}(i)-\bar{A} \geq 0)$ & 0.314 & 0.339 & 0.290 & 0.244 \\
s.d. & 0.16 & 0.20 & 0.17 & 0.21 \\
Obs. & 120 & 120 & 116 & 114 \\
\hline \hline
\end{tabular}

Notes: Average for 15 cases where $c>0$.

and T4 does set optimality increase significantly as the set gets larger. Percentage shares below and above the optimality sets are, on average, quite similar.

Table 13 presents evidence of satisficing, which requires only $\bar{P}(i) \geq \bar{A}$ for $c=0$ separately for treatments T3 and T4 and for $i$ choices yielding $\bar{P}(i)=1$ when satisficing cannot be violated and $\bar{P}(i)<1$. In the latter case, satisficing is predominant but increasing across phases only in T3. The average amount of "burning money", $\phi(\bar{P}(i)-\bar{A})$, is larger for $\bar{P}(i)=1$ than for $\bar{P}(i)<1$ when satisficing, corresponding to $c>0$ behavior. Only for T4 "burning money" is consistently reduced across phases.

Altogether the strongest support for optimality applies under the following conditions: expected utility maximization and set-optimal satisficing coincide, as $c=0$ renders intrapersonal payoff aggregation irrelevant and both concepts are set valued $(e>2)$ rather than point valued $(e=2)$. In fact, for $e>2$, the shares of non-optimal investment choices lie between 5 and 10\% ()see Figure 8). 


\section{Conclusion}

Our analysis assesses the following:

- (sub)optimality across four different between-subject treatments, T1, T2, T3 and T4; two phases with $18 i$ choices each, and all $36 i$ choices of each individual participant; and

- (non)optimal satisficing across T3 and T4, allowing the testing of satisficing and measurement of non-optimal satisficing via "burning money" and "committing suicide" separately for binary random events, boom and doom.

Due to our focus on (sub)optimality and (non)optimal satisficing, our assessments and conclusions are based on robust findings for all cases, both phases and comparable treatments. Neither optimality concept conditions its predictions on probability. What is elicited instead, namely, $\hat{p}$

in $\mathrm{T} 2$ and $\bar{A}$, as well as $\underline{A}$ in $\mathrm{T} 3$ and T4, might cognitively crowd out concentration on behaving (set)optimally but does not affect the (set)optimal choice prediction, $i$.

Assessing and testing (sub)optimality and (non)optimal satisficing for 18 different cases with systematically varying parameters $c, e$ and $p$ and varying optimal investment shares is clearly superior to concentrating on one or two cases whose conclusions could be rather case specific.

Furthermore, optimality in choosing $i$, as well as in aspiration formation, requires rather weak assumptions, namely, Assumption 1 and 2, respectively 3 to prefer $€ 14$ over $€ 4$, respectively to maximize payoffs via repeated use of the slider alerting participants to the payoff consequences of $i$ choices.

Even such rather weak assumptions are systematically violated. This throughly questions accounting for empirical, i.e., experimentally observed, behavior via "rationalizing" with small noise. Neither rationality in making mistakes nor aversion concepts, of which there are several, are applicable in our setup. The systematic rejection of even weak assumptions renders any rational choice explanation an as-if explanation without any claim to be able to explain how choices are generated, i.e., without any behavioral or, specifically, psychological appeal. Nevertheless, one could learn a considerable amount from as if-explanations such as most of our findings; they suggest when and 
why deviations from (set)optimality occur. To provide such a rationalization is, however, quite difficult, since many instruments are ruled out by design.

In our view, taken together, the main findings and statistical results question the view that through consequentialist deliberations we aim at the best outcome. Participants do not generate their choices by anticipating their expected consequences, which presupposes that they are not only fully aware but also certain of the implications of their choice. We often seem to not trust ourselves, and we doubt whether we mentally perceive choice tasks and assess choice consequences correctly.

Our analysis is not merely destructive. Although participants are apparently not "born satisficers", learning and advising could reduce the extent of "burning money" and "committing suicide": suboptimality and non-optimal satisficing may be avoided or reduced when alerting participants to their excessive losses and advising them on how to reduce these losses. Teaching and learning could help limit suboptimality and non-optimal satisficing.

This study should not prevent us from adopting psychological approaches (except for prospect theory and its variants) that view forward-looking consequentialist decision making as a dynamic deliberation process (see for such a process framework, albeit very far from offering an algorithm, Güth and Ploner, 2016), which denies exogenously given preferences and beliefs. A follow-up study will focus on how participants have reacted to specific parameters captured by the 18 different cases, as well as how they responded to what has been elicited in addition to the $i$ choices. This will hopefully provide stylized facts and some orientation when theorizing about what matters, as well as how and when, in (experiments on) risky decision making.

\section{References}

Buchanan, J., and Kock, N., Information overload: A decision making perspective. In Multiple Criteria Decision Making in the New Millennium, 49-58 (Springer Berlin Heidelberg, 2001).

Camerer, C.F., The process-performance paradox in expert judgment: How can experts know so much and predict so badly? in K. A. Ericsson, and J. Smith, eds.: Towards a General Theory of Expertise: Prospects and Limits (Cambridge University Press, New York, 1991). 
Cho, I.K., and Kreps, D.M., "Signaling games and stable equilibria," The Quarterly Journal of Economics, 102 (1987), 179-221.

Fechner, G.T., Vorschule der aesthetik (Vol. 1) (Breitkopf and Härtel, 1976).

Fischbacher, U., "z-Tree: Zurich toolbox for ready-made economic experiments." Experimental economics 10 (2007), 171-178.

Gary, M.S., and Wood, R.E., "Mental models, decision rules, and performance heterogeneity," Strategic Management Journal, 32 (2011), 569-594.

Gilboa, I., and Schmeidler, D., A theory of case-based decisions (Cambridge University Press: 2001).

Güth, W., Levati, M.V., and Ploner, M., "An experimental analysis of satisficing in saving decisions," Journal of Mathematical Psychology, 53 (2009), 265-272.

Güth, W., and Ploner, M., "Mentally perceiving how means achieve ends," mimeo (2016).

Greiner, B., "Subject pool recruitment procedures: organizing experiments with ORSEE." Journal of the Economic Science Association 1(2015), 114-125.

Harless, D.W., and Camerer, C.F., " The predictive utility of generalized expected utility theories," Econometrica, 62 (1994), 1251-1289.

Hey, J.D., "Experimental investigations of errors in decision making under risk," European Economic Review, 39 (1995), 633-640.

Hey, J.D., and Orme, C., "Investigating generalizations of expected utility theory using experimental data," Econometrica, 62 (1994), 1291-1326.

Krantz, D.H., and Kunreuther, H.C., "Goals and plans in decision making," Judgment and Decision Making, 2 (2007), 137-168. 
Kruglanski, A.W., "Goals as knowledge structures," In P. M. Gollwitzer and J. A. Bargh (Eds.) The psychology of action: Linking cognition and motivation to behavior, 599-619 (Guilford Press, New York: 1996).

Kruglanski, A.W., Shah, J.Y., Fishbach, A., Friedman, R., and Chun, W.Y., "A theory of goal systems," In M.P. Zanna (Ed.) Advances in experimental social psychology, 331-378 (Academic Press, San Diego: 2002).

Loomes, G., and Sugden, R., "Incorporating a stochastic element into decision theories," European Economic Review, 39 (1995), 641-648.

McKelvey, R.D., and Palfrey, T.R., "Quantal response equilibria in normal form games," Games and Economic Behaviour, 7 (1995), 6-38.

Marley, A.A.J., "Probabilistic choice as a consequence of nonlinear (sub) optimization," Journal of mathematical psychology, 41 (1997), 382-391.

Myerson, R., "Refinements of the Nash equilibrium concept," International Journal of Game Theory, 7 (1978), 73-80.

Pearl, J., "Causality: models, reasoning and inference," Econometric Theory, 19 (2003), 675-685.

Sauermann, H., and Selten, R., "Anspruchsanpassungstheorie der Unternehmung," Zeitschrift fü die Gesamte Staatswissenschaft, 118 (1962), 577-597.

Savikhin, A.C., "The Application of Visual Analytics to Financial Decision-Making and Risk Management: Notes from Behavioural Economics," In Financial Analysis and Risk Management, 99-114 ( Springer Berlin Heidelberg: 2013).

Selten, R., Pittnauer, S., and Hohnisch, M., "Dealing with dynamic decision problems when knowledge of the environment is limited: an approach based on goal systems," Journal of Behavioral Decision Making, 25 (2012), 443-457.

Selten, R., Sadrieh, A., and Abbink, K., "Money Does Not Induce Risk Neutral Behavior, but Binary Lotteries Do even Worse," Theory and Decision, 46 (1999), 213-252. 
Simon, H.A., "A behavioral model of rational choice," The Quarterly Journal of Economics, 69 (1955), 99-118. 


\section{Appendix A}

In this appendix, we report the translated version of the instructions given to participants. 


\section{CESARE}

\section{INSTRUCTIONS FOR THE EXPERIMENT}

(Not part of Instructions: Treatment 1)

Welcome to our experiment!

Please, read the instructions carefully.

During this experiment you will be asked to make several decisions. These decisions as well as random events will determine your earning. We will now explain the experiment and the payment mechanism.

The experiment consists of two identical phases of 18 rounds each. At the beginning of each round you are endowed with an amount of money that can be allocated in two kinds of investment: investment $\mathrm{A}$ and investment $\mathrm{B}$. Investment $\mathrm{A}$ is a risk-free bond with constant repayment factor, independent of the market condition; Investment $B$ is a risky asset whose repayment factor changes with the market condition and the amount invested in it.

The market can be in good or bad conditions whose probabilities are communicated in each round.

At the end of the experiment, the computer will randomly select a round and you will be paid for that round.

Once the experiment has been completed, you will be asked to answer a questionnaire whose information will be strictly reserved and will be used only anonymously and for research purposes.

Please, work in silence and do not disturb other participants. If you have some doubts, please, raise your hand and wait: one experimenter will come and help you as soon as she can.

ENJOY! 


\section{INVESTMENT CHOICE}

In each round you will be endowed with an amount of money (e), which varies from round to round, that can be allocated between investment A and investment B, by moving the cursor in the bar (see screenshot). Investment $\mathrm{A}$ has a constant repayment factor (c), independent of the market condition; Investment B is risky and its repayment factor changes with market condition (good or bad) and the amount invested: specifically, the investment in B is lost in bad market condition and repays only in good market condition.

Market is in good or bad condition with known probabilities, $\mathbf{p}$ respectively (1-p), which will be communicated in each round.

We will illustrate the choice task in the following example. This will help to familiarize with the screenshots for the investment decisions (see the figure below).

Assume that in a given round you are endowed with $\mathbf{e}=\mathbf{3}$. You must choose how much to invest in the risky asset B (i) and how much to invest in the risk-free bond A (e-i).

The repayment factor for the risk-free bond $\mathrm{A}$ is 0.29 (c).

The repayment factor for the risky asset B depends on the amount you invest (i) and on the market condition, good or bad, whose probabilities, in the example, are 14\% (p) and 86\% (1-p) respectively. In particular, the repayment factor of the risky investment $\mathrm{i}$ is (e-i) in good market condition and $O$ in bad market condition.

Given the endowment (in the example, e=3) and the repayment factor of the risk-free bond (in the example, $c=0.29$ ), your investment in the risky asset (in the example, $i=1$ ) and your investment in the risk-free bond (in the example, $(\mathrm{e}-\mathrm{i})=2)$ will determine your probability of earning $€ 14$ and the complementary probability of earning $€ 4$, which both depend on good or bad market condition, as will be shortly explained.

\section{Please, note that your choice will affect only the probability of earning $€ 14$ or $€ 4$.}

In the good market condition, the probability of earning $€ 14$ is given by the sum of repayment of the risk-free investment, $c^{*}(\mathrm{e}-\mathrm{i})$, and the repayment of the risky investment, (e-i)*i. In the screenshot, the probability of earning $€ 14$, calculated by the computer, is represented by the height of the column corresponding to the good market condition. In the example, given the investment choice of $\mathrm{i}=1$ in the risky asset and (e-i)=2 in the risk-free bond, the probability of earning $€ 14$ is approximately $80 \%$. 
In the bad market condition, the probability of earning $€ 14$ is given only by the repayment of the riskfree investment, $c^{*}(\mathrm{e}-\mathrm{i})$. In the example, the probability of earning $€ 14$, calculated by the computer, is represented by the height of the column corresponding to the bad market condition. In the example, given the investment choice of $\mathrm{i}=1$ in the risky asset and $(\mathrm{e}-\mathrm{i})=2$ in the risk-free bond, the probability of earning $€ 14$ is approximately $50 \%$.

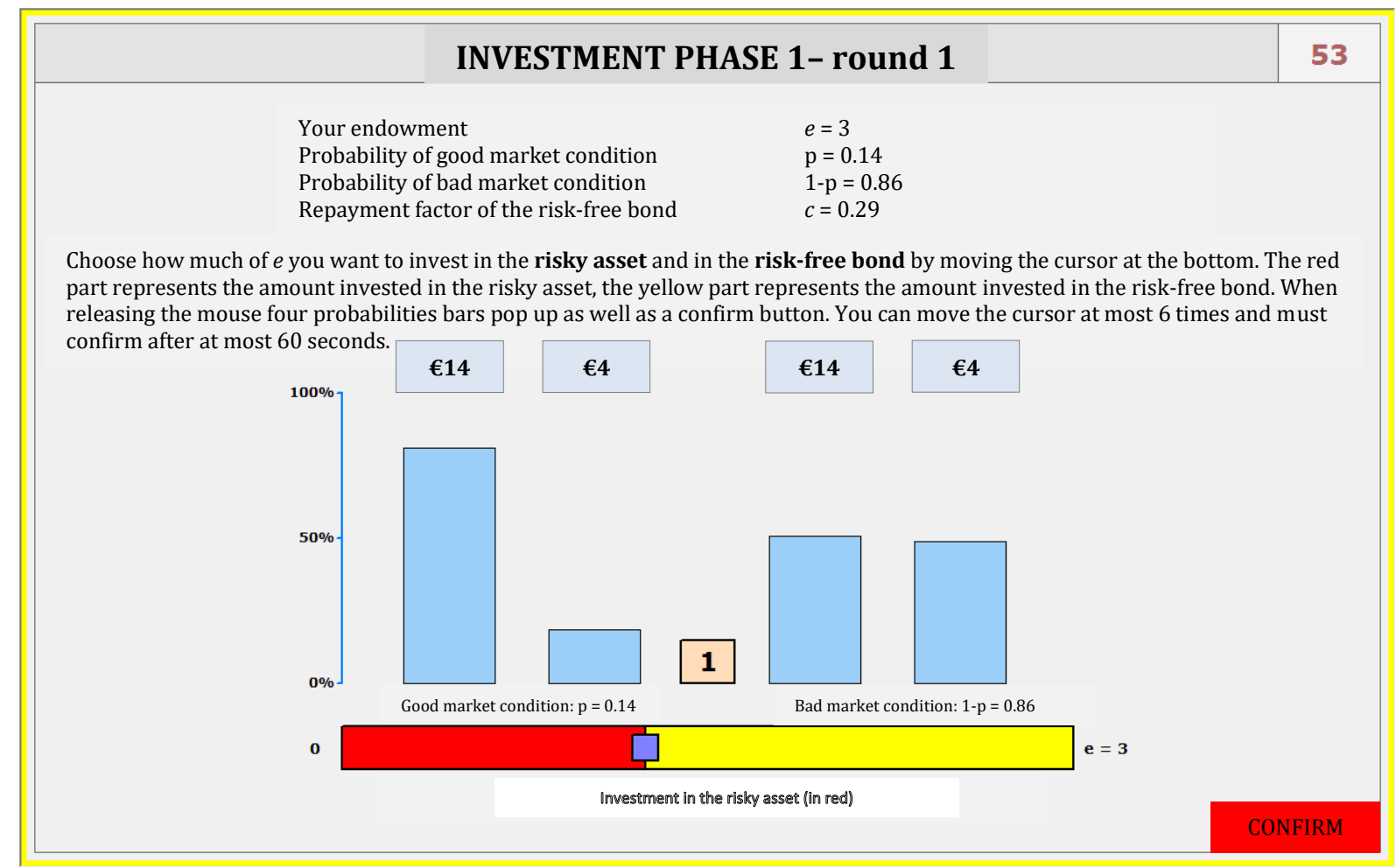

Before making your final decision on how much to invest in the risky asset (i) you can try and visualize the effects of your choice on the probability of earning $€ 14$ in the two different market conditions by scrolling the cursor on the bar. You have several attempts before your final one. You can move the cursor at most 6 times. You can, of course, confirm also an earlier try. The count of your attempts is shown in the centre of the screen by the number between the two columns (in the example, 1 try). The number in the upper right corner up, instead, shows the time elapsed in the current round: for each round, you have at most 60 seconds to make your final choice (in the example, you still have 53 seconds left).

Warning: if you do not confirm your investment choice in time, your last choice before the time expiration will be considered. 


\section{YOUR EARNING IN THE EXPERIMENT}

As already explained, in this experiment you can earn either $€ 14$ or $€ 4$. Your actual earning will depend on the randomly chosen payment round, on your investment decision and on the market condition in that round.

In particular, at the end of the experiment the earning of $\mathbf{€ 1 4}$ or $€ \mathbf{4}$ will be selected by the computer with a probability that depends on:

1. the round randomly chosen for the final payment;

2. your investment choice in that round;

3. the market condition in that round (either good or bad).

In addition, you will receive a show up fee of $€ 4$.

The total earning will be paid individually, privately and immediately after the experiment to each participant. 


\section{CESARE}

\section{INSTRUCTIONS FOR THE EXPERIMENT}

(Not part of Instructions: Treatment 2)

Welcome to our experiment!

Please, read the instructions carefully.

During this experiment you will be asked to make several decisions. These decisions as well as random events will determine your earning. We will now explain the experiment and the payment mechanism.

The experiment consists of two identical phases of 18 rounds each. At the beginning of each round you are endowed with an amount of money that can be allocated in two kinds of investment: investment A and investment B. Investment A is a risk-free bond with constant repayment factor, independent of the market condition; Investment $B$ is a risky asset whose repayment factor changes with the market condition and the amount invested in it.

The market can be in good or bad conditions whose probabilities are unknown. Before the investment choice, you will be asked to state your expectations regarding the probabilities of market in good or bad conditions.

At the end of the experiment, the computer will randomly select a round and you will be paid for that round.

Once the experiment has been completed, you will be asked to answer a questionnaire whose information will be strictly reserved and will be used only anonymously and for research purposes.

Please, work in silence and do not disturb other participants. If you have some doubts, please, raise your hand and wait: one experimenter will come and help you as soon as she can.

\section{ENJOY!}




\section{INVESTMENT CHOICE}

In each round you will be endowed with an amount of money (e), which varies from round to round, that can be allocated between investment $\mathrm{A}$ and investment B, by moving the cursor in the bar (see screenshot). Investment $\mathrm{A}$ has a constant repayment factor (c), independent of the market condition; Investment B is risky and its repayment factor changes with market condition (good or bad) and the amount invested: specifically, the investment in B is lost in bad market condition and repays only in good market condition.

Market is in good or bad condition with unknown probabilities.

We will illustrate the choice task by the following example. This will help to familiarize with the screenshots for the investment decisions (see the figure below).

Assume that in a given round you are endowed with $\mathbf{e}=\mathbf{3}$. You must choose how much to invest in the risky asset B (i) and how much to invest in the risk-free bond A (e-i).

The repayment factor for the risk-free bond $\mathrm{A}$ is 0.29 (c).

The repayment factor for the risky asset B depends on the amount you invest (i) and on the market condition, good or bad. In particular, the repayment factor of the risky investment i is e-i in good market condition and $O$ in bad market condition.

Given the endowment (in the example, e=3) and the repayment factor of the risk-free bond (in the example, $c=0.29$ ), your investment in the risky asset (in the example, $i=1$ ) and your investment in the risk-free bond (in the example, $(e-i)=2)$ will determine your probability of earning $€ 14$ and the complementary probability of earning $€ 4$, which both depend on good or bad market condition, as will be shortly explained.

\section{Please, note that your choice will affect only the probability of earning $€ 14$ or $€ 4$.}

In the good market condition, the probability of earning $€ 14$ is given by the sum of repayment of the risk-free investment, $c^{*}(e-i)$, and the repayment of the risky investment, (e-i)*i. In the screenshot, the probability of earning $€ 14$, calculated by the computer, is represented by the height of the column corresponding to the good market condition. In the example, given the investment choice of $\mathrm{i}=1$ in the risky asset and (e-i)=2 in the risk-free bond, the probability of earning $€ 14$ is approximately $80 \%$.

In the bad market condition, the probability of earning $€ 14$ is given only by the repayment of the risk-

free investment, $\mathrm{c}^{*}(\mathrm{e}-\mathrm{i})$. In the screenshot, the probability of earning $€ 14$, calculated by the computer, 
is represented by the height of the column corresponding to the bad market condition. In the example, given the investment choice of $\mathrm{i}=1$ in the risky asset and (e-i) $=2$ in the risk-free bond, the probability of earning $€ 14$ is approximately $50 \%$.

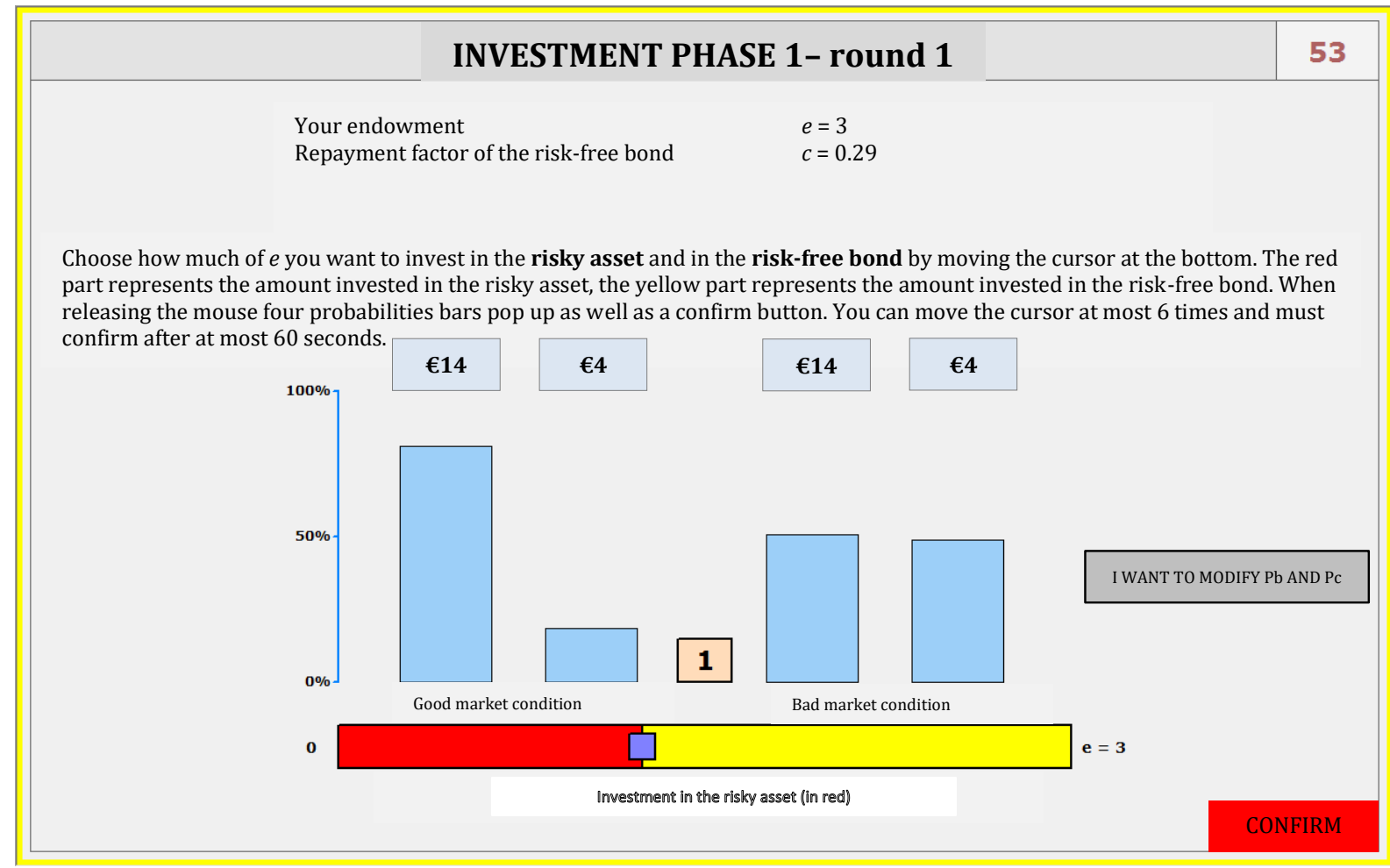

Before making your final decision on how much to invest in the risky asset (i) you can try and visualize the effects of your choice on the probability of earning $€ 14$ in the two different market conditions by scrolling the cursor on the bar. You have several attempts before your final one. You can move the cursor at most 6 times. You can, of course, confirm also an earlier try. The count of your attempts is shown in the centre of the screen by the number between the two columns (in the example, 1 try). The number in the upper right corner up, instead, shows the time elapsed in the current round: for each round, you have at most 60 seconds to make your final choice (in the example, you still have 53 seconds left).

Warning: if you do not confirm your investment choice in time, your last choice before the time expiration will be considered. 
Before the investment choice, in each round you will be asked to state your expectations about the market conditions. In particular, you will be asked to state your expectation regarding the market in good condition and the computer will automatically generate the complementary probability for the market in bad condition. 


\section{STATEMENT OF THE EXPECTATIONS ABOUT MARKET CONDITIONS}

In each round, before your investment choice, you have to state your expectations regarding the probabilities of market in good or bad condition, as shown in the screenshot.

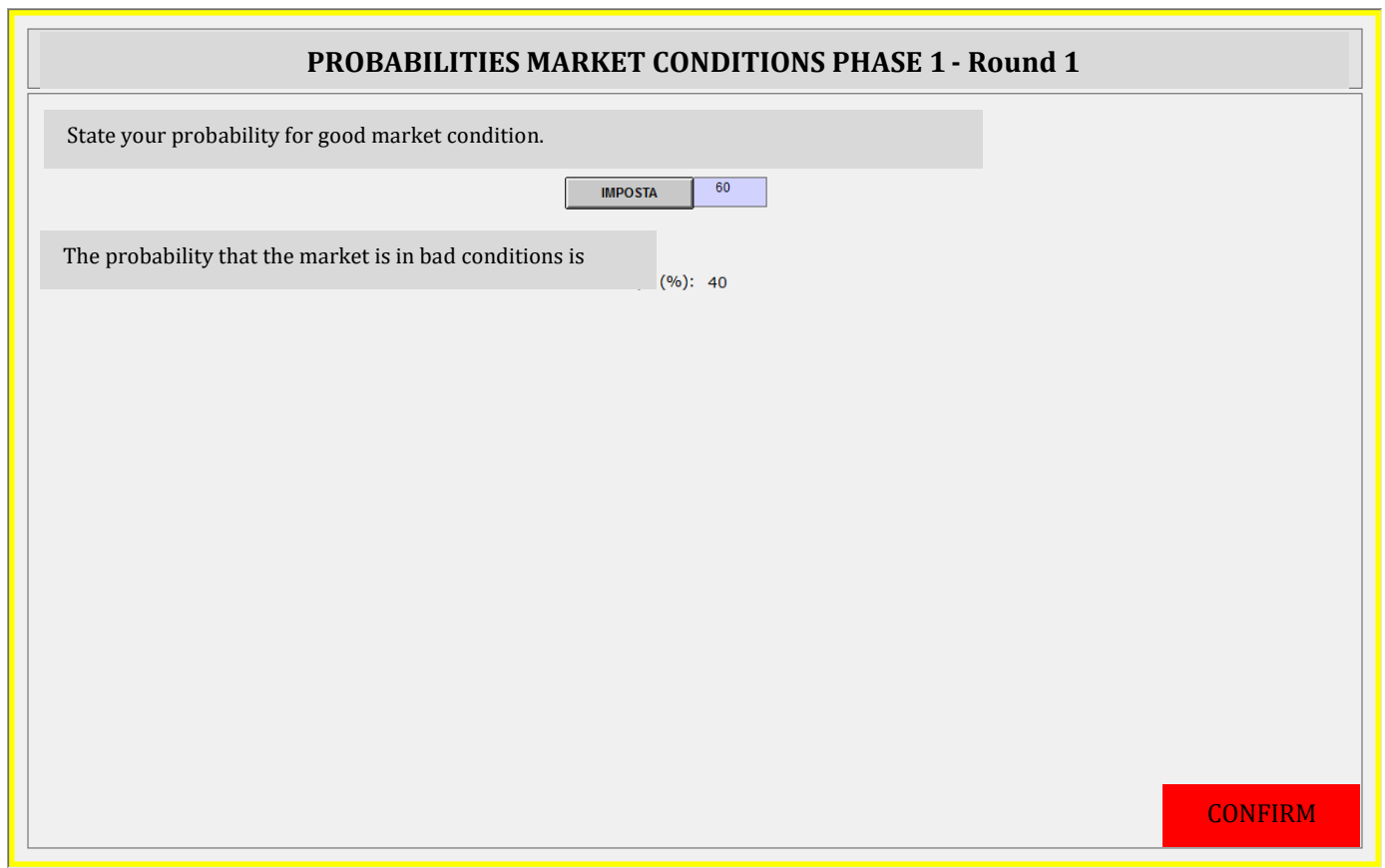

When inserting the probability for good market condition, clicking on "IMPOSTA" the computer automatically generates the complementary probability for bad market condition. By clicking on "Confirm", in the lower right corner of the screen, you can then proceed with your investment choice. 


\section{YOUR EARNING IN THE EXPERIMENT}

As already explained, in this experiment you can earn either $€ 14$ or $€ 4$. Your actual earning will depend on the randomly chosen payment round, on your investment decision and on the market condition in that round.

In particular, at the end of the experiment the earning of $\mathbf{€ 1 4}$ or $€ \mathbf{4}$ will be selected by the computer with a probability that depends on:

1. the round randomly chosen for the final payment;

2. your investment choice in that round;

3. the market condition in that round (either good or bad).

In addition, you will receive a show up fee of $€ 4$.

The total earning will be paid individually, privately and immediately after the experiment to each participant. 


\section{CESARE}

\section{INSTRUCTIONS FOR THE EXPERIMENT}

(Not part of Instructions: Treatment 3)

Welcome to our experiment!

Please, read the instructions carefully.

During this experiment you will be asked to make several decisions. These decisions as well as random events will determine your earning. We will now explain the experiment and the payment mechanism.

The experiment consists of two identical phases of 18 rounds each. At the beginning of each round you are endowed with an amount of money that can be allocated in two kinds of investment: investment $\mathrm{A}$ and investment $\mathrm{B}$. Investment $\mathrm{A}$ is a risk-free bond with constant repayment factor, independent of the market condition; Investment $B$ is a risky asset whose repayment factor changes with the market condition and the amount invested in it.

The market can be in good or bad conditions whose probabilities are communicated in each round.

At the end of the experiment, the computer will randomly select a round and you will be paid for that round.

Once the experiment has been completed, you will be asked to answer a questionnaire whose information will be strictly reserved and will be used only anonymously and for research purposes.

Please, work in silence and do not disturb other participants. If you have some doubts, please, raise your hand and wait: one experimenter will come and help you as soon as she can.

ENJOY! 


\section{INVESTMENT CHOICE}

In each round you will be endowed with an amount of money (e), which varies from round to round, that can be allocated between investment A and investment B, by moving the cursor in the bar (see screenshot). Investment $\mathrm{A}$ has a constant repayment factor (c), independent of the market condition; Investment B is risky and its repayment factor changes with market condition (good or bad) and the amount invested: specifically, the investment in B is lost in bad market condition and repays only in good market condition.

Market is in good or bad condition with known probabilities, $\mathbf{p}$ respectively (1-p), which will be communicated in each round.

We will illustrate the choice task by the following example. This will help to familiarize with the screenshots for the investment decisions (see the figure below).

Assume that in a given round you are endowed with $\mathbf{e}=\mathbf{3}$. You must choose how much to invest in the risky asset B (i) and how much to invest in the risk-free bond A (e-i).

The repayment factor for the risk-free bond $\mathrm{A}$ is 0.29 (c).

The repayment factor for the risky asset B depends on the amount you invest (i) and on the market condition, good or bad, whose probabilities, in the example, are 14\% (p) and 86\% (1-p) respectively. In particular, the repayment factor of the risky investment $\mathrm{i}$ is (e-i) in good market condition and $O$ in bad market condition.

Given the endowment (in the example, e=3) and the repayment factor of the risk-free bond (in the example, $c=0.29$ ), your investment in the risky asset (in the example, $i=1$ ) and your investment in the risk-free bond (in the example, $(\mathrm{e}-\mathrm{i})=2)$ will determine your probability of earning $€ 14$ and the complementary probability of earning $€ 4$, which both depend on good or bad market condition, as will be shortly explained.

\section{Please, note that your choice will affect only the probability of earning $€ 14$ or $€ 4$.}

In the good market condition, the probability of earning $€ 14$ is given by the sum of repayment of the risk-free investment, $c^{*}(\mathrm{e}-\mathrm{i})$, and the repayment of the risky investment, (e-i)*i. In the screenshot, the probability of earning $€ 14$, calculated by the computer, is represented by the height of the column corresponding to the good market condition. In the example, given the investment choice of $\mathrm{i}=1$ in the risky asset and (e-i)=2 in the risk-free bond, the probability of earning $€ 14$ is approximately $80 \%$. 
In the bad market condition, the probability of earning $€ 14$ is given only by the repayment of the riskfree investment, $c^{*}(\mathrm{e}-\mathrm{i})$. In the example, the probability of earning $€ 14$, calculated by the computer, is represented by the height of the column corresponding to the bad market condition. In the example, given the investment choice of $\mathrm{i}=1$ in the risky asset and $(\mathrm{e}-\mathrm{i})=2$ in the risk-free bond, the probability of earning $€ 14$ is approximately $50 \%$.

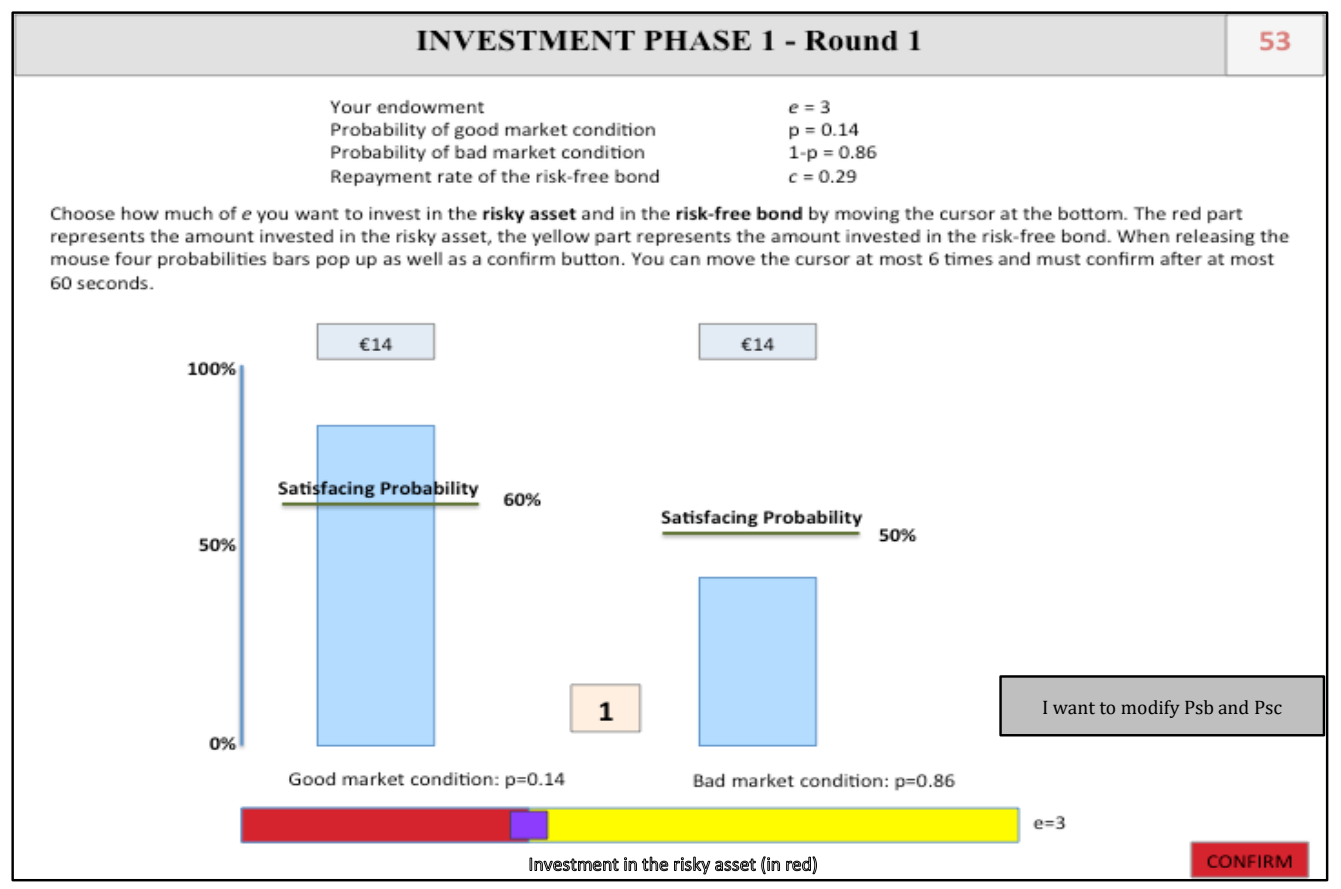

Before making your final decision on how much to invest in the risky asset (i) you can try and visualize the effects of your choice on the probability of earning $€ 14$ in the two different market conditions by scrolling the cursor on the bar. You have several attempts before your final one. You can move the cursor at most 6 times. You can, of course, confirm also an earlier try. The count of your attempts is shown in the centre of the screen by the number between the two columns (in the example, 1 try). The number in the upper right corner up, instead, shows the time elapsed in the current round: for each round, you have at most 60 seconds to make your final choice (in the example, you still have 53 seconds left).

Warning: if you do not confirm your investment choice in time, your last choice before the time expiration will be considered. 
Before the investment choice, in each round you will be asked to state the probability of earning $€ 14$ instead of $€ 4$ that would make you satisfied, both for market in good and bad condition separately. You will earn $€ 14$ with the probability that makes you satisfied if the probability implied by your investment choice $\mathrm{i}$ (the height of $€ 14$-column in the screenshot) is not smaller than the probability that makes you satisfied (in the screenshot your stated satisficing probability is shown by the horizontal line). On the contrary, if the probability implied by your investment choice $\mathrm{i}$ (the height of the $€ 14$-column in the screenshot) is smaller than the probability that makes you satisfied, you will earn $€ 14$ with $0 \%$ probability. 


\section{STATEMENT OF THE PROBABILITY OF EARNING €14 THAT MAKES YOU SATISFIED}

In each round, before your investment choice, you will have to state the probability of earning $€ 14$ instead of $€ 4$ that would make you satisfied for both markets in good condition $\left(\mathrm{P}_{\mathrm{sb}}\right)$ and in bad condition $\left(\mathrm{P}_{\mathrm{sc}}\right)$, separately.

Warning: the probability that makes you satisfied for the market in good condition cannot be smaller than the probability for the market in bad condition.

In the example below $\mathrm{P}_{\mathrm{sb}}$ is set at $60 \%$ and $\mathrm{P}_{\mathrm{sc}}$ is set at $50 \%$.

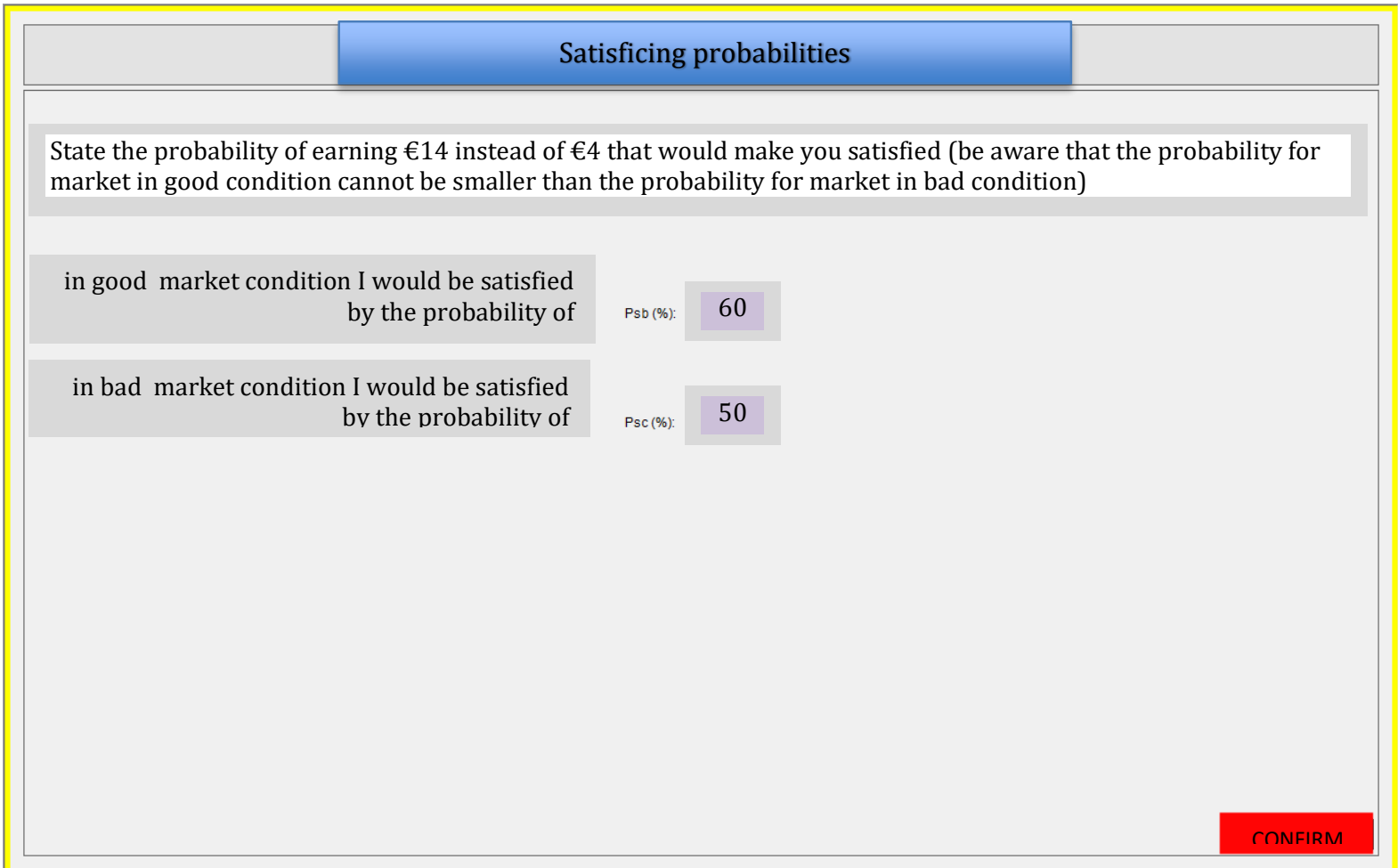

By clicking on "Confirm", in the lower right corner of the screen, you can then proceed with your investment choice.

Warning: you can modify the probability that makes you satisfied only once, by clicking on the button "I want to modify $\mathrm{P}_{\mathrm{sb}}$ and $\mathrm{P}_{\mathrm{sc}}$ ". 


\section{YOUR EARNING IN THE EXPERIMENT}

As already explained, in this experiment you can earn either $€ 14$ or $€ 4$. Your actual earning will depend on the randomly chosen payment round, on your investment decision and on the market condition in that round and on your stated aspiration levels.

In particular, at the end of the experiment the earning of $\mathbf{€ 1 4}$ or $€ \mathbf{4}$ will be selected by the computer with a probability that depends on:

1. the round randomly chosen for the final payment;

2. your investment choice in that round;

3. the market condition in that round (either good or bad);

4. whether the probability of earning $€ 14$ that makes you satisfied is achieved in the following way:

a) If the probability of earning $€ 14$ that makes you satisfied is achieved, the probability of earning $€ 14$ is equal to your satisficing probability for the market condition of the round randomly chosen.

In the example, the market in good condition and the investment choice $i=1$ determine $a$ probability of $80 \%$ of earning $€ 14$. Since the stated satisficing probability for the market in good condition is $60 \%$, the result is the following:

- You achieved the stated satisficing probability of 60\%;

- You earn $€ 14$ with probability $60 \%$ and $€ 4$ with probability $40 \%$.

b) If the probability of earning $€ 14$ that makes you satisfied is not achieved, the probability of earning $€ 14$ is $0 \%$.

In the example, in case of market in bad condition and the investment choice $i=1$, the result is the following:

- You earn $€ 14$ with probability 0\%, which means that you earn $€ 4$ with probability $100 \%$.

In addition, you will receive a show up fee of $€ 4$.

The total earning will be paid individually, privately and immediately after the experiment to each participant. 


\section{CESARE}

\section{INSTRUCTIONS FOR THE EXPERIMENT}

(Not part of Instructions: Treatment 4)

Welcome to our experiment!

Please, read the instructions carefully.

During this experiment you will be asked to make several decisions. These decisions as well as random events will determine your earning. We will now explain the experiment and the payment mechanism.

The experiment consists of two identical phases of 18 rounds each. At the beginning of each round you are endowed with an amount of money that can be allocated in two kinds of investment: investment A and investment B. Investment A is a risk-free bond with constant repayment factor, independent of the market condition; Investment $B$ is a risky asset whose repayment factor changes with the market condition and the amount invested in it.

The market can be in good or bad conditions whose probabilities are unknown.

At the end of the experiment, the computer will randomly select a round and you will be paid for that round.

Once the experiment has been completed, you will be asked to answer a questionnaire whose information will be strictly reserved and will be used only anonymously and for research purposes.

Please, work in silence and do not disturb other participants. If you have some doubts, please, raise your hand and wait: one experimenter will come and help you as soon as she can.

ENJOY! 


\section{INVESTMENT CHOICE}

In each round you will be endowed with an amount of money (e), which varies from round to round, that must be allocated between investment A and investment B, by moving the cursor in the bar (see screenshot). Investment $\mathrm{A}$ has a constant repayment factor (c), independent of the market condition; Investment B is risky and its repayment factor changes with market condition (good or bad) and the amount invested: specifically, the investment in B is lost in bad market condition and repays only in good market condition.

Market is in good or bad condition with unknown probabilities.

We will illustrate the choice task by an example. This will help to familiarize with the screenshots for the investment decisions (see the figure below).

Assume that in a given round you are endowed with $\mathbf{e}=\mathbf{3}$. You must choose how much to invest in the risky asset B (i) and how much to invest in the risk-free bond A (e-i).

The repayment factor for the risk-free bond $\mathrm{A}$ is 0.29 (c).

The repayment for the risky asset B depends on the amount you invest (i) and on the market condition, good or bad. In particular, the repayment factor of the risky investment i is e-i in good market condition and $O$ in bad market condition.

Given the endowment (in the example, 3) and the repayment factor of the risk-free bond (in the example, 0.29), your investment in the risky asset $(i)$ and your investment in the risk-free bond (e-i) will determine your probability of earning $€ 14$ and the complementary probability of earning $€ 4$, which both depend on good or bad market condition.

\section{Please, note that your choice will affect only the probability of earning $€ 14$ or $€ 4$.}

In the good market condition, the probability of earning $€ 14$ is given by the sum of repayment of the risk-free investment, $c^{*}(e-i)$, and the repayment of the risky investment, (e-i)*i. In the screenshot, the probability of earning $€ 14$, calculated by the computer, is represented by the height of the column corresponding to the good market condition. In the example, given the investment choice of $i=1$ in the risky asset and $(e-i)=2$ in the risk-free bond, the probability of earning $€ 14$ is approximately $80 \%$.

In the bad market condition, the probability of earning $€ 14$ is given only by the repayment of the riskfree investment, $c^{*}(e-i)$. In the example, the probability of earning $€ 14$, calculated by the computer, is represented by the height of the column corresponding to the bad market condition. In the example, 
given the investment choice of $\mathrm{i}=1$ in the risky asset and $(\mathrm{e}-\mathrm{i})=2$ in the risk-free bond, the probability of earning $€ 14$ is approximately $50 \%$.

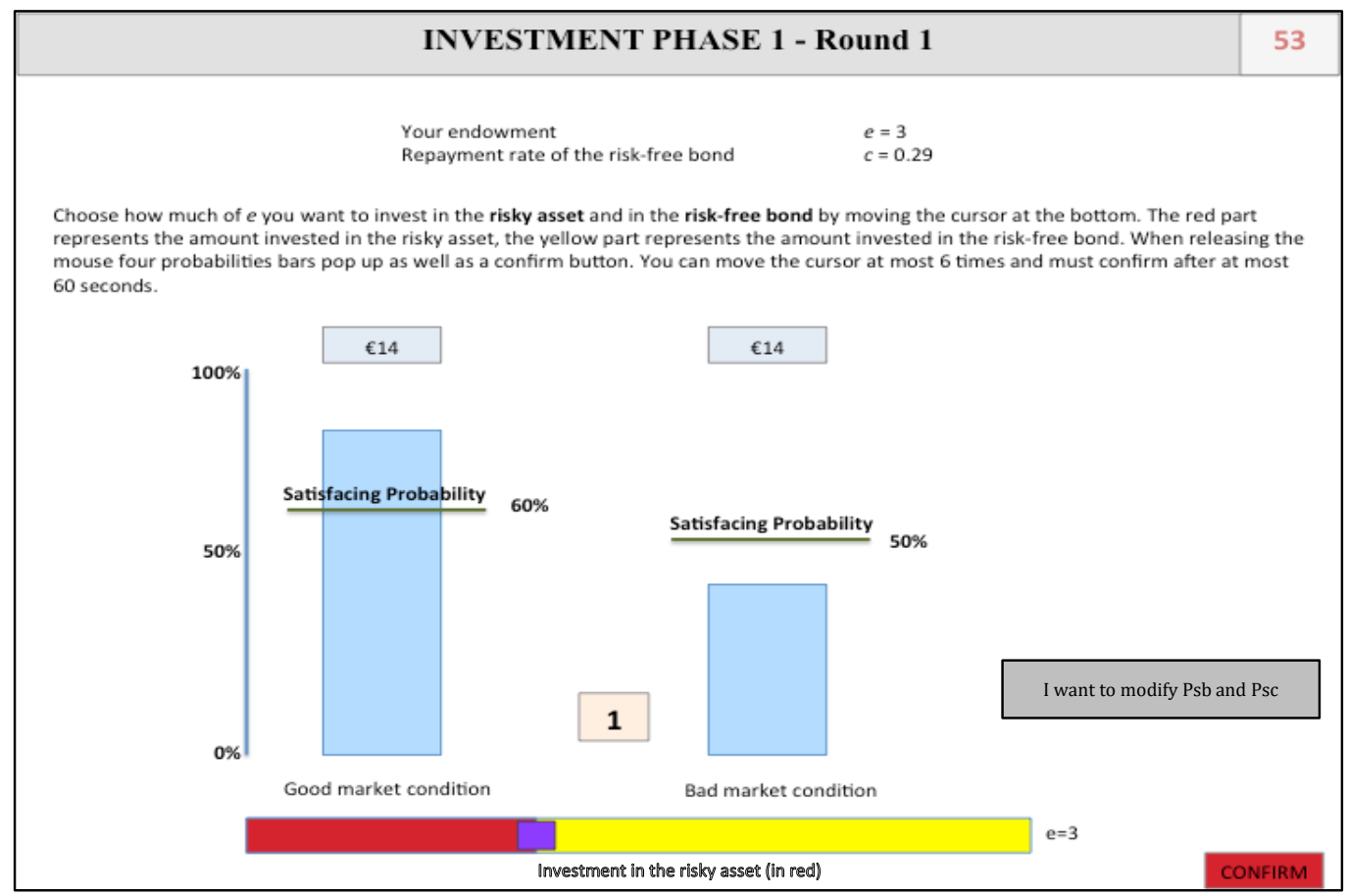

Before making your final decision on how much to invest in the risky asset $(i)$ you can try and visualize the effects of your choice on the probability of earning $€ 14$ in the two different market conditions by scrolling the cursor on the bar. You have several attempts before your final one. You can move the cursor at most 6 times. You can, of course, confirm also an earlier try. The count of your attempts is shown in the centre of the screen by the number between the two columns (in the example, 1 try). The number in the upper right corner up, instead, shows the time elapsed in the current round: for each round, you have at most 60 seconds to make your final choice (in the example, you still have 53 seconds left).

Warning: if you do not confirm your investment choice in time, your last choice before the time expiration will be considered.

Before the investment choice, in each round you will be asked to state the probability of earning $€ 14$ instead of $€ 4$ that would make you satisfied, both for market in good and bad condition separately. 
You will earn $€ 14$ with the probability that makes you satisfied if the probability implied by your investment choice $\mathrm{i}$ (the height of $€ 14$-column in the screenshot) is not smaller than the probability that makes you satisfied (in the screenshot your stated satisficing probability is shown by the horizontal line). On the contrary, if the probability implied by your investment choice i (the height of the $€ 14$-column in the screenshot) is smaller than the probability that makes you satisfied, you will earn $€ 14$ with $0 \%$ probability. 


\section{STATEMENT OF THE PROBABILITY OF EARNING €14 THAT MAKES YOU SATISFIED}

In each round, before your investment choice, you will have to state the probability of earning $€ 14$ instead of $€ 4$ that would make you satisfied for both markets in good condition $\left(\mathrm{P}_{\mathrm{sb}}\right)$ and in bad condition $\left(\mathrm{P}_{\mathrm{sc}}\right)$, separately.

Warning: the probability that makes you satisfied for the market in good condition cannot be smaller than the probability for the market in bad condition.

In the example below $\mathrm{P}_{\mathrm{sb}}$ is set at $60 \%$ and $\mathrm{P}_{\mathrm{sc}}$ is set at $50 \%$.

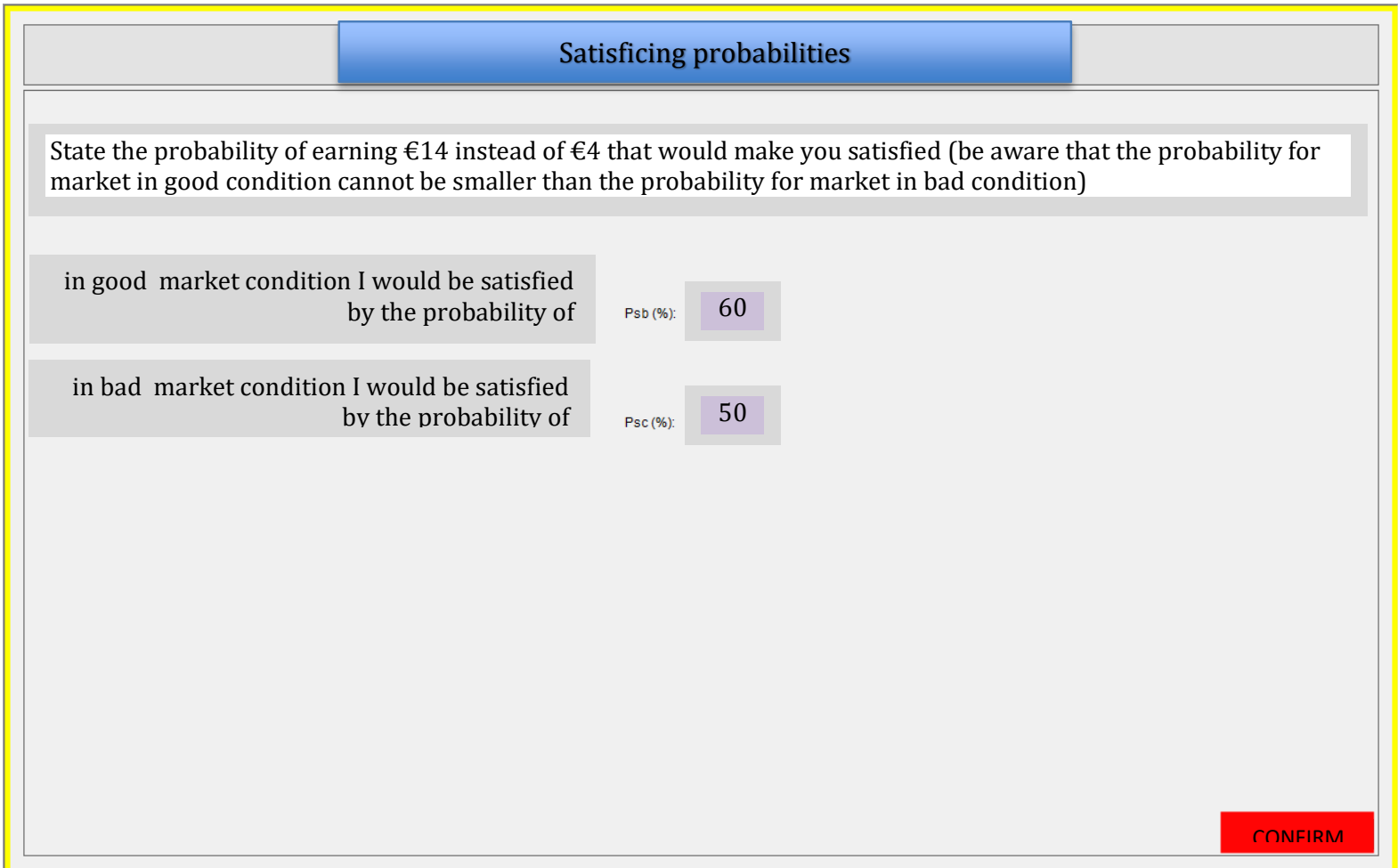

By clicking on "Confirm", in the lower right corner of the screen, you can then proceed with your investment choice.

Warning: you can modify the probability that makes you satisfied only once, by clicking on the button "I want to modify $\mathrm{P}_{\mathrm{sb}}$ and $\mathrm{P}_{\mathrm{sc}}$ ". 


\section{YOUR EARNING IN THE EXPERIMENT}

As already explained, in this experiment you can earn either $€ 14$ or $€ 4$. Your actual earning will depend on the randomly chosen payment round, on your investment decision and on the market condition in that round.

In particular, at the end of the experiment the earning of $\mathbf{€ 1 4}$ or $€ \mathbf{4}$ will be selected by the computer with a probability that depends on:

1. the round randomly chosen for the final payment;

2. your investment choice in that round;

3. the market condition in that round (either good or bad);

4. whether the probability of earning $€ 14$ that makes you satisfied is achieved in the following way:

a) If the probability of earning $€ 14$ that makes you satisfied is achieved, the probability of earning $€ 14$ is equal to your satisficing probability for the market condition of the round randomly chosen.

In the example, the market in good condition and the investment choice $i=1$ determine $a$ probability of $80 \%$ of earning $€ 14$. Since the stated satisficing probability for the market in good condition is $60 \%$, the result is the following:

- You achieved the stated satisficing probability of 60\%;

- You earn $€ 14$ with probability $60 \%$ and $€ 4$ with probability $40 \%$.

b) If the probability of earning $€ 14$ that makes you satisfied is not achieved, the probability of earning $€ 14$ is $0 \%$.

In the example, in case of market in bad condition and the investment choice $i=1$, the result is the following:

- You earn $€ 14$ with probability 0\%, which means that you earn $€ 4$ with probability $100 \%$.

In addition, you will receive a show up fee of $€ 4$.

The total earning will be paid individually, privately and immediately after the experiment to each participant. 


\section{Appendix B}

Figure 9: Investment choice in the experiment

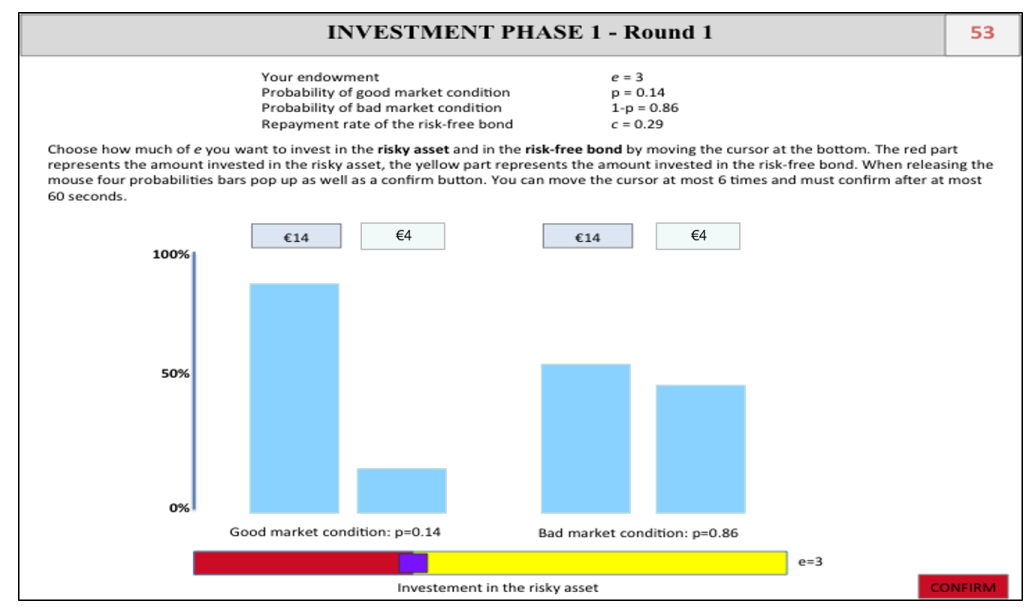

Figure 10: Investment choice in the experiment

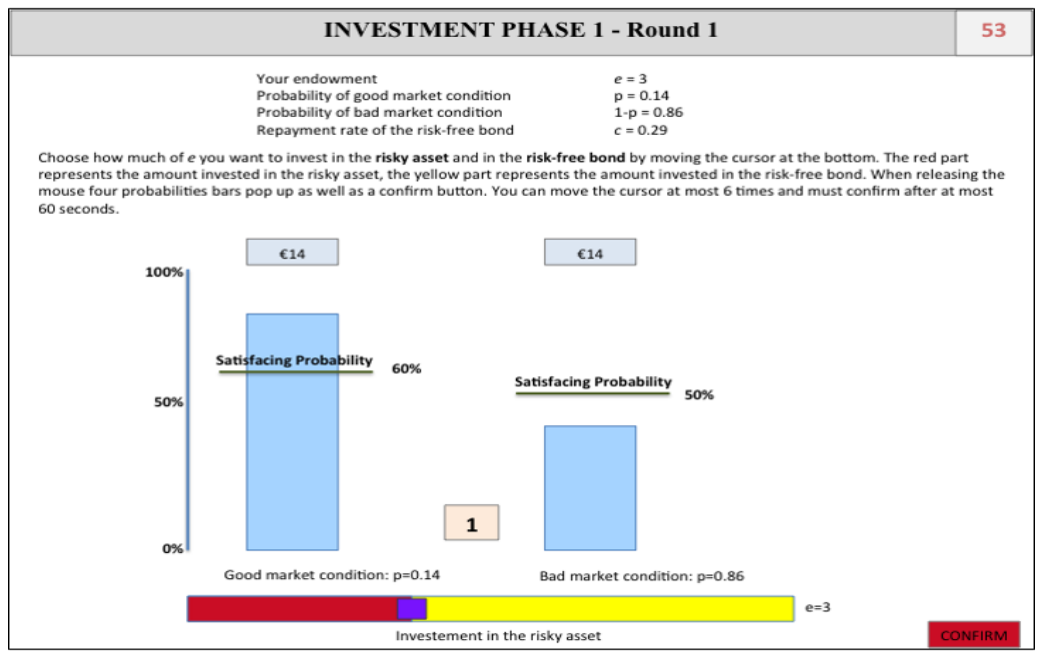


Figure 11: Deviation $i^{\circ}-i$ by Phase
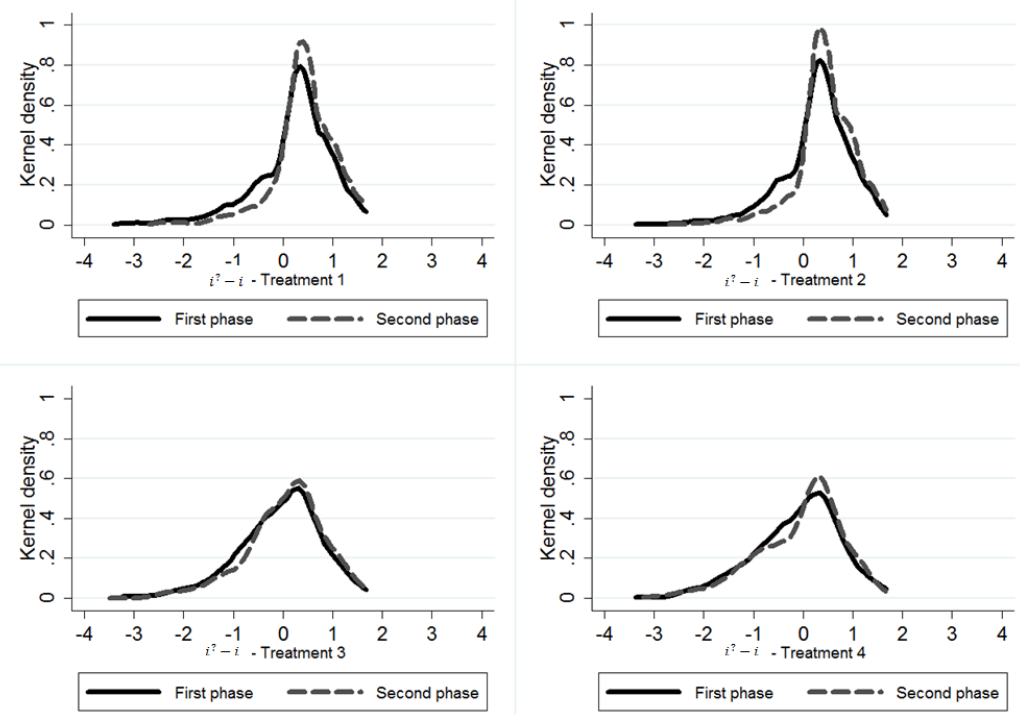

Notes: Kernel density function for cases where $c>0$; Treatment 2 investment $i^{\circ}$ has been computed based on objective probabilities $p$.

Figure 12: Distance in payoff space: $\bar{P}\left(i^{*}\right)-\bar{P}(i)$ by phase (boom cases)
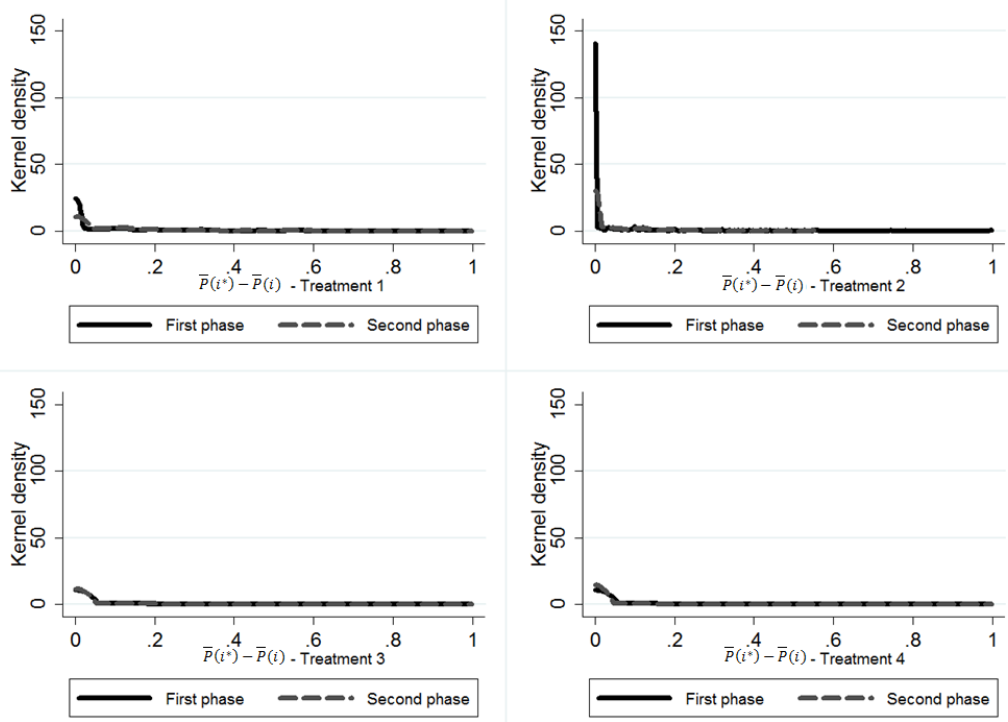

Notes: Kernel density function considering only cases where $c>0$. 
Figure 13: Distance in payoff space: $\underline{P}\left(i^{*}\right)-\underline{P}(i)$ by phase (doom cases)
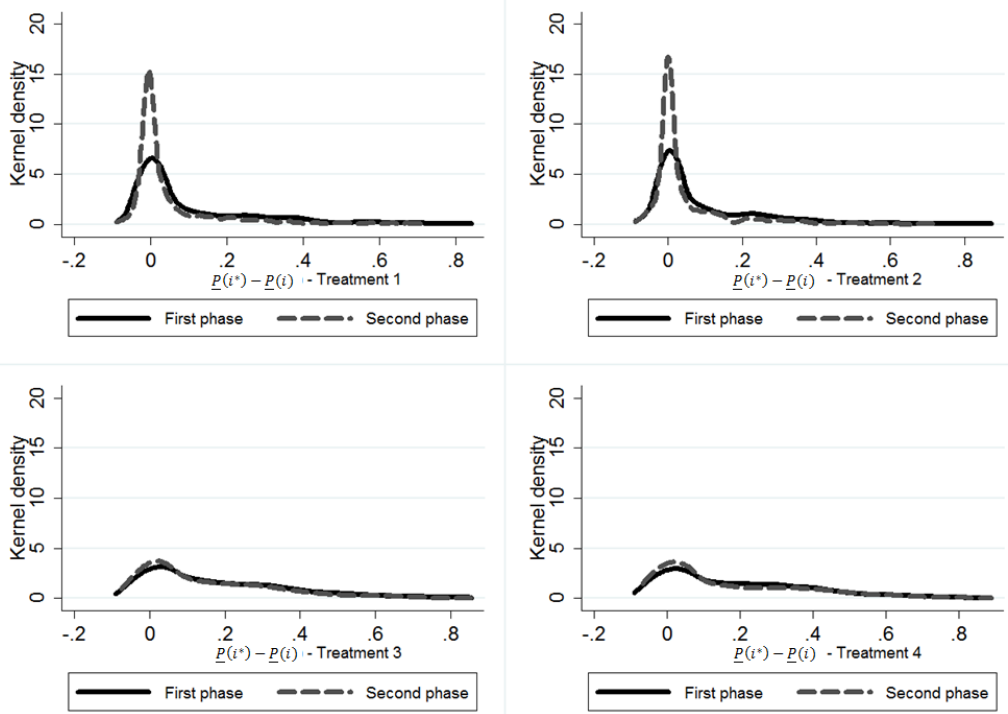

Notes: Kernel density function considering only cases where $c>0$.

Table 14: Difference tests for probability $p$ and probability $\hat{p}$ and payoff space deviation - T1 vs. T2

\begin{tabular}{l|ccc}
\hline \hline & & \multicolumn{3}{|c}{ T1 vs. T2 } \\
& $p$ in T1 & $\hat{p}$ in T2 & WRST Ho: Prob(T1) $=\operatorname{Prob}(\mathrm{T} 2)$ \\
\hline Phase 1 & 0.289 & 0.555 & 0.00 \\
Phase 2 & 0.289 & 0.539 & 0.00 \\
WRST Ho: Prob(Ph1) $=\operatorname{Prob}(\mathrm{Ph} 2)$ & 1.00 & 0.08 & \\
\hline \hline
\end{tabular}

Notes: The analysis refers to Wilcoxon Rank Sum Test (WRST) for two independent samples.

Table 15: Action and payoff space comparison - T1 vs. T4

\begin{tabular}{l|ccc|cccc|ccccc}
\hline \hline & \multicolumn{4}{|c}{$i^{*}-i$} & \multicolumn{4}{c}{$P\left(i^{*}\right)-P(i)$} & $\bar{P}\left(i^{*}\right)-\bar{P}(i)$ & \multicolumn{4}{c}{$\underline{P}\left(i^{*}\right)-\underline{P}(i)$} \\
\hline T1 vs. T4 & T1 & T4 & WRST(Ind.) & T1 & T4 & WRST & T1 & T4 & WRST & T1 & T4 & WRST \\
\hline All sample & -0.305 & -0.718 & $0.000(0.000)$ & 0.076 & 0.152 & 0.000 & 0.063 & 0.030 & 0.000 & 0.075 & 0.175 & 0.000 \\
Phase 1 & -0.409 & -0.749 & $0.000(0.000)$ & 0.095 & 0.161 & 0.000 & 0.056 & 0.035 & 0.000 & 0.102 & 0.185 & 0.000 \\
Phase 2 & -0.201 & -0.688 & $0.000(0.000)$ & 0.057 & 0.142 & 0.000 & 0.071 & 0.025 & 0.000 & 0.049 & 0.165 & 0.000 \\
KST(Ind.) & $0.000(0.000)$ & $0.000(0.000)$ & & 0.000 & 0.000 & & 0.000 & 0.842 & & 0.000 & 0.000 & \\
\hline \hline
\end{tabular}

Notes: A Wilcoxon Rank Sum Test (WRST) for two independent samples was performed for comparison across treatments, and a Kolmogorov-Smirnov equality of distributions test (KST) was performed for the analysis across phases. We report the tests for individual averages in parentheses. For these tests, p-values are reported. 
Table 16: Action and payoff space comparison - T2 vs. T4

\begin{tabular}{l|ccc|cccc|ccc|cc}
\hline \hline & \multicolumn{4}{|c}{$i^{*}-i$} & \multicolumn{4}{c}{$P\left(i^{*}\right)-P(i)$} & \multicolumn{1}{c}{$\left(i^{*}\right)-\bar{P}(i)$} & \multicolumn{4}{c}{$\underline{P}\left(i^{*}\right)-\underline{P}(i)$} \\
\hline T2 vs. T4 & T2 & T4 & WRST(Ind.) & T2 & T4 & WRST & T2 & T4 & WRST & T2 & T4 & WRST \\
\hline All sample & -0.282 & -0.718 & $0.000(0.000)$ & 0.070 & 0.152 & 0.000 & 0.043 & 0.030 & 0.000 & 0.069 & 0.175 & 0.000 \\
Phase 1 & -0.369 & -0.749 & $0.000(0.000)$ & 0.087 & 0.161 & 0.000 & 0.040 & 0.035 & 0.000 & 0.091 & 0.185 & 0.000 \\
Phase 2 & -0.195 & -0.688 & $0.000(0.000)$ & 0.054 & 0.142 & 0.000 & 0.045 & 0.025 & 0.000 & 0.046 & 0.165 & 0.000 \\
KST(Ind.) & $0.000(0.000)$ & $0.000(0.000)$ & & 0.000 & 0.000 & & 0.000 & 0.842 & & 0.000 & 0.000 \\
\hline \hline
\end{tabular}

Notes: A Wilcoxon Rank Sum Test (WRST) for two independent samples was performed for comparison across treatments, and a Kolmogorov-Smirnov equality of distributions test (KST) was performed for the analysis across phases. We report the tests for individual averages in parentheses. For these tests, p-values are reported. In T2, $P\left(i^{*}\right)$ is computed by objective probabilities $p$.

Figure 14: Deviations from optimal aspirations $\bar{P}(i)-\bar{A}$ for given $i$ choices and $\underline{P}(i)-\underline{A}$ by Treatment
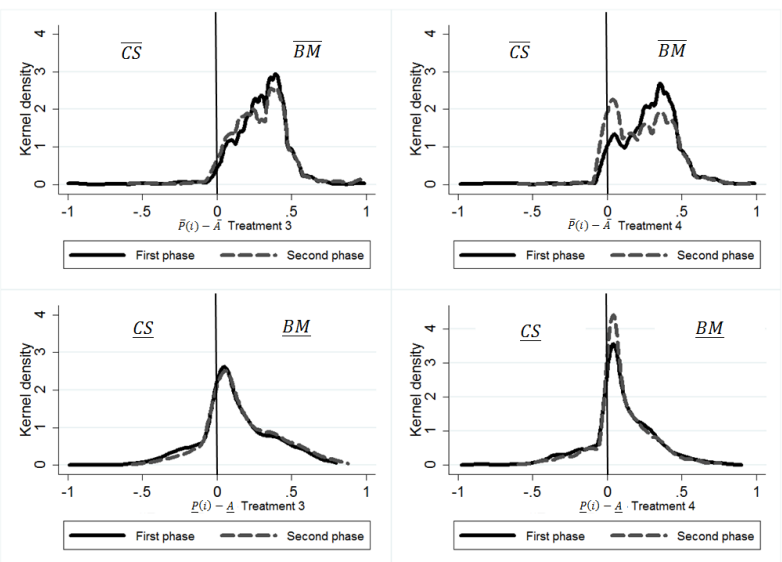

Notes:Kernel density function.

Table 17: $B M_{\epsilon}$ and $C S_{\epsilon}$ comparison - T3 vs. T4

\begin{tabular}{|c|c|c|c|c|c|c|c|c|c|c|c|c|}
\hline & \multicolumn{3}{|c|}{$\overline{\overline{B M}} \bar{M}_{\epsilon}$} & \multicolumn{3}{|c|}{$\underline{B M_{\epsilon}}$} & \multicolumn{3}{|c|}{$\overline{C S_{\epsilon}}$} & \multicolumn{3}{|c|}{$\underline{C S_{\epsilon}}$} \\
\hline T3 vs. T4 & T3 & T4 & WRST & T3 & T4 & WRST & T3 & T4 & WRST & T3 & T4 & WRST \\
\hline Phase 1 & 0.318 & 0.326 & 0.343 & 0.272 & 0.226 & 0.000 & 0.401 & 0.493 & 0.237 & 0.226 & 0.239 & 0.823 \\
\hline Obs. & 1055 & 936 & & 663 & 594 & & 27 & 20 & & 188 & 175 & \\
\hline Phase 2 & 0.322 & 0.315 & 0.932 & 0.291 & 0.213 & 0.000 & 0.282 & 0.239 & 0.846 & 0.198 & 0.216 & 0.248 \\
\hline Obs. & 1031 & 806 & & 714 & 591 & & 19 & 11 & & 136 & 134 & \\
\hline KST & 0.022 & 0.005 & & 0.264 & 0.366 & & 0.112 & 0.022 & & 0.144 & 0.526 & \\
\hline \multirow[t]{2}{*}{ T3 vs. T4 } & T3 & T4 & "WRST & $\overline{\mathrm{T} 3}$ & T4 & 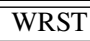 & $\overline{\mathrm{T} 3}$ & $\overline{\mathrm{T} 4}$ & "WRST & T3 & $\overline{\mathrm{T} 4}$ & WRST \\
\hline & & $\overline{B M}$ & & & $\underline{B M}$ & & & $\overline{C S}$ & & & $\underline{C S}$ & \\
\hline Phase 1 & 0.309 & 0.310 & 0.974 & 0.212 & $\overline{0.165}$ & 0.000 & 0.351 & 0.369 & 0.932 & 0.172 & $\overline{0.202}$ & 0.060 \\
\hline Obs. & 1087 & 988 & & 871 & 855 & & 31 & 27 & & 254 & 210 & \\
\hline Phase 2 & 0.308 & 0.273 & 0.000 & 0.231 & 0.149 & 0.000 & 0.212 & 0.152 & 0.390 & 0.139 & 0.173 & 0.015 \\
\hline Obs. & 1085 & 941 & & 922 & 864 & & 26 & 18 & & 201 & 171 & \\
\hline KST & 0.003 & 0.000 & & 0.271 & 0.070 & & 0.020 & 0.031 & & 0.064 & 0.275 & \\
\hline
\end{tabular}

Notes: A Wilcoxon Rank Sum Test (WRST) for two independent samples was performed for comparison across treatments, and a Kolmogorov-Smirnov equality of distributions test (KST) was performed for the analysis across phases. Cases with $c=0$ are excluded. The first part of the table considers the misspecification $\epsilon$, while the second part does not. 